\title{
Comparison of gut microbiota between adults with autism spectrum disorder and obese adults
}

\author{
Qiang Zhang ${ }^{1,2}$, Rong Zou ${ }^{2}$, Min Guo ${ }^{2}$, Mengmeng Duan ${ }^{2}$, Quan Li ${ }^{\text {Corresp., } 1}$, Huajun Zheng ${ }^{\text {Corresp. } 2}$ \\ ${ }^{1}$ Department of Obstetrics and Gynecology, Affiliated Hospital of Zunyi Medical University, Zunyi, China \\ 2 NHC Key Lab. of Reproduction Regulation (Shanghai Institute of Planned Parenthood Research), Fudan University, Shanghai, China \\ Corresponding Authors: Quan Li, Huajun Zheng \\ Email address: 1013283493@qq.com, zhenghj@chgc.sh.cn
}

Background: Autism spectrum disorder (ASD) and obesity are serious global public health problems. Studies have shown that ASD children are at a higher risk of obesity than the general population. To investigate the gut microbe characteristics of adults ASD and obese adults, we compared the gut microbiota of adults with ASD to obese adults. Methods: The fecal samples were collected from 21 adult patients with ASD and 21 obese adults, and V3V4 regions of $16 \mathrm{~S}$ rRNA genes were sequenced by high-throughput DNA sequencing. The gut microbiota of adults with ASD and obese adults was compared. Results: We observed the proportion of Firmicutes/Bacteroidetes in ASD was significantly increased, with families Lachnospiraceae and Ruminococcaceae significantly enriched in adult ASD. Eighteen genera, including Lachnospiracea incertae sedis, Ruminococcus, Blautia, and Holdemanella were significantly increased in adult ASD, whereas Megamonas and Fusobacterium were significantly increased in obesity. At the species level, we found six species enriched in ASD and three species enriched in obesity, including Phascolarctobacterium succinatuten producing propionate. Dialister succinatiphilus may be as a biomarker for predicting obesity, as well as Prevotella copri may be a common-owned pathogens of ASD and obesity. Conclusions: Some conflicting results have been reported in microbiota studies of ASD, which may be related to age and obesity. Thus, the body mass index should be evaluated before analyzing the gut microbiota of patients with ASD, as obesity is prevalent in these individuals and gut microbiota is severally affected by obesity. 


\section{Comparison of gut microbiota between adults with} 3 autism spectrum disorder and obese adults

4

5

6 Qiang Zhang ${ }^{1,2}$, Rong Zou ${ }^{2}$, Min Guo ${ }^{2}$, Mengmeng Duan², Quan $\mathrm{Li}^{1 *}$, Huajun Zheng²* 7

$8{ }^{1}$ Department of Obstetrics and Gynecology, Affiliated Hospital of Zunyi Medical University, 9 Zunyi, Guizhou, China.

2 NHC Key Lab. of Reproduction Regulation (Shanghai Institute of Planned Parenthood Research), Fudan University, Shanghai, China.

\section{*Corresponding Authors:}

Quan $\mathrm{Li}^{1}$

No. 149, Dalian Road, Zunyi, Guizhou, 563000, China.

Email address: 1013283493@qq.com

Huajun Zheng ${ }^{2}$

No. 2140, Xietu Road, Shanghai, 200032, China.

Email address: zhenghj@chgc.sh.cn

\section{Abstract}

Background: Autism spectrum disorder (ASD) and obesity are serious global public health problems. Studies have shown that ASD children are at a higher risk of obesity than the general population. To investigate the gut microbe characteristics of adults ASD and obese adults, we compared the gut microbiota of adults with ASD to obese adults. 
26 Methods: The fecal samples were collected from 21 adult patients with ASD and 21 obese

27 adults, and V3-V4 regions of 16S rRNA genes were sequenced by high-throughput DNA

28 sequencing. The gut microbiota of adults with ASD and obese adults was compared.

29 Results: We observed the proportion of Firmicutes/Bacteroidetes in ASD was significantly

30 increased, with families Lachnospiraceae and Ruminococcaceae significantly enriched in

31 adult ASD. Eighteen genera, including Lachnospiracea incertae sedis, Ruminococcus, Blautia,

32 and Holdemanella were significantly increased in adult ASD, whereas Megamonas and

33 Fusobacterium were significantly increased in obesity. At the species level, we found six

34 species enriched in ASD and three species enriched in obesity, including

35 Phascolarctobacterium succinatuten producing propionate. Dialister succinatiphilus may be

36 as a biomarker for predicting obesity, as well as Prevotella copri may be a common-owned

37 pathogens of ASD and obesity.

38 Conclusions: Some conflicting results have been reported in microbiota studies of ASD, 39 which may be related to age and obesity. Thus, the body mass index should be evaluated 40 before analyzing the gut microbiota of patients with ASD, as obesity is prevalent in these 41 individuals and gut microbiota is severally affected by obesity.

Keywords: autism spectrum disorder; obesity; gut microbiota; adult autism spectrum disorder; 16S rRNA

\section{Introduction}

47 Autism spectrum disorder (ASD) is a group of complex neurodevelopmental disorders 48 characterized by persistent deficits in social reciprocity and verbal/nonverbal social interaction 49 communicative behaviors, as well as the presence of repetitive and restricted patterns of 50 behaviors, activities, or interests (American Psychiatric Association, 2013). According to the 51 report of World Health Organization (WHO) (https://www.who.int/news-room/fact- 
52 sheets/detail/autism-spectrum-disorders), ASD begins before the age of 3 and persists throughout 53 a person's life, with an average of one in 160 children worldwide suffering from ASD. There are 54 great differences in intelligence of individuals with ASD (American Psychiatric Association, 55 2013), and most of them need lifelong care from family and society, which greatly influence patients' physical and mental health as well as socio-economic development. Therefore, ASD is a serious global public health problem.

Accumulating evidence has indicated that ASD children are at a higher risk of obesity than the general population (Dhaliwal et al., 2019; Healy, Aigner \& Haegele, 2019; Hill, Zuckerman \& Fombonne, 2015; Zheng et al., 2017), and autistic adults were more likely to be overweight or obese than non-autistic people (Sedgewick, Leppanen \& Tchanturia, 2020). Obesity is a complex metabolic disease with unclear etiology, which usually defined as according to body mass index (BMI). In adults, obesity is defined as a BMI of $\geq 30 \mathrm{~kg} / \mathrm{m}^{2}$ (Bray et al., 2018; Jensen et al., 2014). According to the report of WHO (https://www.who.int/zh/news-room/fact-sheets/detail/obesity-and-overweight), in 2016, more than 650 million adults were obese. With increasing prevalence of obesity, the risk of obesityassociated diseases such as cardiovascular disease, stroke, type 2 diabetes, hypertension, nonalcoholic fatty liver disease, and some types of cancer is increasing (Jensen et al., 2014; Ogden et al., 2014). Thus, obesity is also a serious public health problem.

In recent decades, increasing attention has been paid to the role of intestinal microbiota on both health and disease. Harmonious symbiosis of intestinal microbiota is the key to maintaining human health. Once the micro-ecological balance is broken, which probably lead to variety disorders, including ASD (Fattorusso et al., 2019) and obesity (Mitev \& Taleski, 2019). Several studies have suggested that the microbiota-gut-brain axis plays a vital role in the occurrence and development of ASD (Luna, Savidge \& Williams, 2016; Martin et al., 2018; van Sadelhoff et al., 2019). The brain can affect the composition of gut microbiota through regulating host intestinal motility, secretion and permeability, and then bring about gastrointestinal 
78

79

80

81

82

83

84

85

86

87

88

89

90

91

92

93

94

95

96

97

98

99

100

101

102

103

symptoms of individuals with ASD (Luna, Savidge \& Williams, 2016; Martin et al., 2018; van Sadelhoff et al., 2019). Moreover, gut microbiota in turn affects the function of central nervous system (CNS) in the host via neurotransmitter, immune, or metabolite products, which can lead to the ASD-like behaviors (MacFabe, 2015; van Sadelhoff et al., 2019).

Additionally, another study has shown that the microbiota-gut-brain axis also plays an important role in the development of obesity (Torres-Fuentes et al., 2017). Intestinal microbiota may be contributed to the occurrence and development of obesity by influencing the host's nutrient metabolism, energy balance, inflammation, and insulin resistance (Khan et al., 2016; Torres-Fuentes et al., 2017). Moreover, intestinal microbiota and its metabolites can directly stimulate the vagus and transmit stimulus signals to the CNS or indirectly act on the CNS through immune-neuroendocrine mechanisms, then affecting the feeding behavior of the body (Fetissov, 2017; Torres-Fuentes et al., 2017). In addition, the CNS can control the feeding behavior of host, and then provide nutrition for intestinal flora or affect the composition of intestinal microbiota (Fetissov, 2017).

Through association between obesity and ASD is often reported, most studies focused on risk factors contributing to obesity, like individuals with ASD often have picking eating behavior, spend less time on physical activities, have comorbidities associated with obesity, etc (Dhaliwal et al., 2019; Zheng et al., 2017). But how does obesity affect ASD has not been reported. Stanislawski et al. reported that a lower alpha diversity and a higher relative abundance of Prevotella are positively correlated with obesity among black and Hispanic populations (Stanislawski et al., 2019). In our study, we also observed that the Prevotella was significantly increased in adults with ASD compared to healthy adults (in press). So we postulate that gut microbiota changes caused by obesity might be a contributing factor affecting ASD development. But comparison of intestinal microbiota characteristics between patients with ASD and obese patients has not been reported. Therefore, in this study, we determined and compared gut microbiota of 21 adult patients with ASD and 21 obese adults, to identify the similarities and 
104 differences of intestinal microbiota between them. Based on this, we expect to provide potential

105 therapies and preventive measures for patients with ASD or obesity.

106

107 Materials and methods

108 Sample collection

109 Twenty-one patients diagnosed with ASD (mean BMI $=22.8,15.9-31.9$, with 6 females and 15

110 males) with ages ranging from 17 to 32 were recruited from the XinWangAi Caring Center for

111 People with Intellectual Disability (Jinan, Shandong Province, China), and their care costs are

112 mainly from social donation and government financial expenditure. These patients with ASD are

113 all Han nationality, and diagnosed in childhood by clinicians according to the diagnostic criteria

114 for childhood autism in International Classification of Diseases and Related Health Problems,

$11510^{\text {th }}$ Revision (ICD-10) (World Health Organization, 1993). Patients with schizophrenia or other

116 psychosis, or having taken antibiotics for one month prior to fecal sample collection were

117 excluded. Most of the 21 ASD patients have gastrointestinal symptoms such as constipation and

118 diarrhea. Twenty-one gender and age matched obese adults (mean BMI $=35.3,31.4-49.6)$ that

119 did not suffer from ASD, other neurodevelopmental disorders or neuropsychiatric diseases, and

120 were not under dietary or medication control to lose weight, were recruited from a gym in Jinan.

121 Stool specimens were collected during the daytime using MicroLocker ${ }^{\mathrm{T}}$ stool sample collector

122 (YM-F02B, JiangSu YIMI Biotech Inc., China) which contains fecal sample preservation

123 solution, and transferred to laboratory within three hours . All samples were stored at $-80^{\circ} \mathrm{C}$ until

124 DNA extraction. The study was approved by the Medical Ethical Committee of Shanghai

125 Institute of Planned Parenthood Research (NO: PJ2019-17). Written informed consent was

126 obtained from the parents/guardians for all participants involved in this study. All methods were

127 performed in accordance with the Declaration of Helsinki.

128

129 Genomic DNA extraction, PCR amplification, and 16S rRNA gene sequencing 
130 DNA extraction and PCR amplification were performed as described previously (Zou et al.,

131 2020). Specially, the fecal DNA was extracted using the QIAamp DNA Stool Mini Kit

132 (QIAGEN, Hilden, Germany). The V3-V4 region of 16S rRNA genes was amplified using

133 primers 338F and 806R (Huse et al., 2007) with TransStart Fastpfu DNA Polymerase (TransGen,

134 Beijing, China) in 20 cycles. Three replicate PCR amplifications of each sample were purified

135 with AxyPrep DNA Gel Extraction kit (AXYGEN, Union City, CA, USA), then pooled into

136 equal concentrations after quantification. Next, $2 \times 300$ paired-end sequencing was performed

137 for the equivalent pooled 16S rRNA PCR amplicons on an Illumina MiSeq instrument (San 138 Diego, CA, USA) .

139

140 Bioinformatics and statistical analysis

141 Sequencing data was analyzed using Mothur (version 1.39.5) (Schloss, Gevers \& Westcott, 2011)

142 as previously described (Zou et al., 2020). In brief, the reads containing ambiguous bases, length

143 shorter than 350 base pairs, with chimeric sequence or contaminant sequence were firstly

144 removed. Then the SILVA reference database (Quast et al., 2013) (V132) was used as a

145 reference for OTU identification under the threshold of 97\% similarity. Community richness,

146 evenness, and diversity were assessed using Mothur. Differences between ASD and obesity

147 samples were assessed by analysis of molecular variance (AMOVA). The taxonomic

148 assignments were based on the Ribosomal Database Project (Cole et al., 2009) with the default

149 parameter $(80 \%$ threshold). Microbiota functions were predicted using phylogenetic

150 investigation of communities by reconstruction of unobserved states (PICRUSt) (Langille et al.,

151 2013). The significant differences in relative abundance of microbial taxa (OTU, genus, family,

152 and phylum) and microbiota functional profiles between the ASD and obese groups were

153 analyzed with STAMP using two-sided Welch's t-test (Parks et al., 2014). The coefficient

154 relationship between species was calculated using $\mathrm{R}$ package with Spearman correlation 
155 algorithm, and the correlation parameters were set as: coefficient $>0.35$ or $<-0.35$ and $p<0.05$

156 (Taylor, 1990).

157

158

Accession numbers

159 The sequence data have been deposited in the National Omics Data Encyclopedia (NODE) under

160 accession

number

OEX010410

161 (https://www.biosino.org/node/review/detail/OEV000113?code=KYM47EZL) and OEX010411

162 (https://www.biosino.org/node/review/detail/OEV000114?code=BS6WW5QC).

163

164 Results

165 Bacterial composition in adult gut of ASD and obese subjects

166 A total of 42 fecal samples were collected from 21 adult patients with ASD and 21 obese adults.

167 A total of 2,039,712 (39,341-59,610) high-quality 16S rRNA genes from 42 samples were

168 contained by high-throughput DNA sequencing. To normalize the data and avoid statistical bias,

16939,341 16S rRNA genes from each sample were chosen to calculate the richness, evenness, and

170 diversity of bacterial community at $97 \%$ similarity. After the 42 samples were classified into two

171 groups (ASD and Obesity), 12,411 operational taxonomic units (OTUs) were obtained

172 (Supplemental Information). The Good's coverage was over 99.8\% in the two groups (Table

173 1), indicating that the sequencing depth was sufficient for studying the gut microbiota in adult

174 individuals with ASD and obese adults.

175

176 Microbiota of $A S D$ and obesity

177 The total gut microbiota was examined by phylogenetic and taxonomic assessments of the 16S

178 rRNA V3-V4 regions. Approximately 99.2\% $( \pm 0.0041)$ of microbiota could be aligned to 18

179 phyla, $96.0 \%( \pm 0.0364)$ to 98 families, and $87.1 \%( \pm 0.0891)$ to 269 genera. At the phylum level,

180 Bacteroidetes (average 48.5\%, \pm 0.221 ), Firmicutes (average 43.6\%, \pm 0.204 ), and Proteobacteria 
181 (average $2.93 \%, \pm 0.051$ ) were the three most abundant bacterial groups in the gut, which were

182 common phyla in all samples (Table 2). At the family level, 15 families showed major 183 abundance in two groups ( $>1 \%$ in at least one group, accounting for over $90 \%$ in each group,

184 Table 3). Among the 15 families, Lachnospiraceae, Prevotellaceae, and Bacteroidaceae were 185 dominant ( $>64 \%$ of each group). In the 269 identified genera, 47 genera were major genera $186(>0.1 \%$ in at least one group), including Bacteroides, Prevotella, Megamonas, Roseburia,

187 Lachnospiracea incertae sedis, Faecalibacterium, and so on (Table 4). Among the major genera, 188 seven ubiquitous (core) genera were consistently found across all analyzed samples and 189 comprised an average of $>23 \%$ of the total microbiota, including Bacteroides, L. incertae sedis, 190 Streptococcus, Ruminococcus 2, Dorea, Blautia, and Clostridium XIVa.

192 Bacterial composition changes between ASD and obese groups

193 Among the 21 adult ASD, five were underweight $(\mathrm{BMI}<18.5)$ and two were obese $(\mathrm{BMI}>30)$. 194 AMOVA analysis revealed that the gut microbiota composition among the three groups (obese 195 ASD, underweight ASD and normal weight ASD) had no significant difference (Table 5, Figure 196 1A), while the whole ASD group showed significant difference with obese group ( $\left.\mathrm{P}_{\mathrm{AmOVA}}<0.05\right)$. 197 Hereinafter, we take all the 21 adult ASD as a whole to compare with obese group. Principal 198 component analysis (Figure 1B) showed that most subjects in the ASD and obese groups were 199 distant from each group based on the gut microbiota composition. According to the evaluation of 200 bacterial populations (Figure 2, Table 1), subjects with ASD showed higher richness (ACE 201 index and Chao index), higher evenness (Shannon even index), and higher diversity (Shannon 202 and Simpson index). Thus, microbiota compositions differed between the ASD and obese groups, 203 with the ASD showing higher biodiversity compared to the obese group.

204 At the phylum level, three major abundance phyla showed no significant variations 205 between the ASD and obese groups. Only the phylum Fusobacteria was significantly decreased 206 in $\operatorname{ASD}(\mathrm{p}=0.031)$ from $3.51 \%$ in the obese group to $0.10 \%$ in the ASD group. At the family 
207 level (Figure 3), seven families showed significant differences between the ASD and obesity 208 groups, six of which were major abundance families. The results showed that ASD was generally 209 associated with the proportions of families. At the genus level (Table 4, Figure 4), 20 genera 210 were found to significantly differ between ASD (16.62\%) and obese groups (19.28\%), 12 of 211 which were major genera. Only two genera were decreased in the ASD group: Megamonas and 212 Fusobacterium.

213 At the species level (OTU from top 50, Table 6), nine abundant species significantly 214 differed between ASD and obese subjects. Three were increased in the obesity gut microbiota, 215 including Megamonas funiformis, Fusobacterium mortiferum, and Dialister succinatiphilus. Six 216 species were increased in the ASD gut microbiota, including Blautia wexlerae, Blautia faecis, 217 Eubacterium eligens, Ruminococcus faecis, Phascolarctobacterium succinatutens, and 218 Holdemanella biformis.

219

220

221

\section{Predicted functional potential change between ASD and obese microbiota}

We used PICRUSt to predict the functional potential changes in ASD and Obesity (Table 7,

Figure 5). Thirty-three pathways differed between ASD and obese subjects, with 30 of which 223 belonging to metabolism and three pathways belonging to environmental information processing. 224

\section{5}

226

227

228

229

230

231

232

\section{Correlations between bacterial species}

To characterize the microbial interactions of ASD gut microbiota, correlation patterns of the top 10 species and different species between the ASD and obese groups were calculated (Table 8, Figure 6, $\mathrm{p}<0.05)$. In the ASD groups, 12 species showed correlations, including eight different species. In the obese group, 13 species showed correlations, including six different species. Four correlated species were shared by ASD and obese microbiota: B. wexlerae, Blautia faecis, G. formicilis, and Bacteroides vulgatus.

Peer) reviewing PDF | (2020:08:52367:2:0:NEW 16 Jan 2021) 


\section{Comparison of normal weight ASD and obesity}

234 If we compare the alpha diversity, we can see that the normal weight ASD group ( $\mathrm{n}=14)$ had

235 lower richness but higher diversity than the whole ASD group $(n=21)$ (Table 1), though this

236 difference had no statistical significance. To exclude the affection of weight on gut microbiota,

237 we then compare the normal weight ASD $(n=14)$ and obese group $(n=21)$.

238 At the phylum level, the increase of phylum Firmicutes and the decrease of phylum

239 Bacteroidetes in the normal weight ASD showed statistical significance, which was not found

240 when comparing all the ASD and obese group (Table 9). At the family level, the same seven

241 families showed significant differences between the normal weight ASD and obesity groups

242 (Table 9). At the genus level (Table 9), 16 genera were found to significantly differ with obese

243 group, with genus Allisonella significantly decreased in the normal weight ASD group in

244 addition Megamonas and Fusobacterium. At the species level, Bacteroides plebeius was

245 significantly decreased in normal weight ASD, which was not observed when taking all the ASD

246 as a whole (Table 9). Meanwhile, the abundance change of Eubacterium eligens and

247 Holdemanella biformis showed no longer significance.

248

249 Discussion

250 To characterize similarities and differences in the microbiota of adults with ASD and obese

251 subjects, taxonomy assignments and difference analysis were performed between the two groups.

252 In this study, we observed seven genera (Bacteroides, Streptococcus, Dorea, L. incertae sedis,

253 Ruminococcus, Blautia, and Clostridium XIVa) with an abundance of $23.75 \%( \pm 0.0199$ in two

254 groups) as core microbiota (Table 4, Figure 3). The microbiota of adults with ASD showed

255 higher biodiversity than in obese control subjects; one phylum, seven families, 20 genera, and 13

256 species significantly differed between the two groups.

257 Previous studies suggested that Bacteroidetes, Proteobacteria, and Fusobacteria were 258 enriched in children with ASD, while Firmicutes and Actinobacteria were lower in ASD (Coretti 
259 et al., 2018; De Angelis et al., 2013; Ma et al., 2019; Zhang et al., 2018). In the present study,

260 though five phyla showed abundance changes of greater than $1 \%$, only the relative abundance of

261 phylum Fusobacteria showed a significant decrease in ASD group $(p<0.05)$ compared to the

262 obese group (Table 2). Consistent with our findings, Andoh et al. reported a relative abundance

263 of the phylum Fusobacteria increased in fecal of adults with obesity compared to lean people

264 (Andoh et al., 2016). The rising Firmicutes/Bacteroidetes (F/B) ratio has been suggested as an

265 indicator of obesity, as Koliada et al. have reported that a higher abundance of Firmicutes and a

266 lower level of Bacteroidetes in adults with obesity than in normal-weight adults in Ukraine

267 (Koliada et al., 2017). But in our study, the proportion of F/B was significantly higher in adults

268 with ASD (1.14) than that in adults with obesity $(0.70)(\mathrm{p}<0.05$, Wilcoxon rank-sum test)

269 (Table 2). Although dietary habits have been proposed to contribute to this ratio difference

270 (Zhang et al., 2018), age may be also involved. Consequently, we conjectured that the proportion

271 of F/B may be closely associated with both ASD and obesity.

272 At the family level, we observed Lachnospiraceae, Ruminococcaceae,

273 Erysipelotrichaceae, Porphyromonadaceae, and Desulfovibrionaceae were enriched in adults

274 with ASD, while Fusobacteriaceae and Veillonellaceae were significantly decreased;

275 Prevotellaceae was dominant family both ASD (28.9\%) and obese (32.5\%) groups (Table 3).

276 Serena et al. have indicated that the families Veillonellaceae and Prevotellaceae were

277 significantly increased in obese individuals compared to healthy subjects (Serena et al., 2018),

278 which are major bacteria succinate-producing (Nakayama et al., 2017). In adipose tissue,

279 succinate possesses antilipolytic actions through binding to cognate receptor succinate receptor 1

280 (Sncr1), and leads to fat accumulation (McCreath et al., 2015). Therefore, Veillonellaceae and

281 Prevotellaceae were playing an important role in development of obesity. Compared with non-

282 obese adults with ASD, we found that a higher abundance

283 of Pseudomonaceae, Prevotellaceae, and Fusobacteriaceae, as well as a lower abundance of

284 Lachnospiraceaea and Ruminococcaceae in fecal of obese adults. These results were consistent 
285 with previous studies on gut microflora in appendix samples of obese patients (Moreno-Indias et

286 al., 2016). As is known, the families Lachnospiraceaea and Ruminococcaceae were able to

287 ferment carbohydrates to produce short-chain fatty acids (SCFAs) which mainly includes acetic 288 acid, propionic acid and butyric acid (Biddle et al., 2013). Among SCFAs, butyrate can inhibit

289 the release of pro-inflammatory cytokines like TNF- $\alpha$ and IL-6 and play an anti-inflammatory

290 role (Lewis et al., 2010).

291 At the genus level, only two genera (Megamonas and Fusobacterium) were significantly 292 decreased, while 18 genera were increased in the ASD group. The abundance of Megamonas 293 decreased from $11.67 \%$ in obese group to only $0.7 \%$ in adults with ASD (Table 4, Figure 4). It

294 had been reported that Megamonas can ferment glucose into acetic and propionic acid, which has

295 been shown to be a substrate for lipogenesis and cholesterol formation and serve as an energy 296 source for the host (Kieler et al., 2017). Consistent with our findings, previous study reported 297 that Megamonas was enriched in obese adults, which was positively associated with obesity 298 (Chiu et al., 2014; Maya-Lucas et al., 2019). Additionally, Andoh et al. have reported that the 299 genus Fusobacterium was significantly enriched in obese individuals compared to lean people 300 (Andoh et al., 2016). The genus Fusobacterium belongs to the phylum Fusobacteria, which may

301 be involved into the occurrence and development of obesity by inducing the host's inflammatory 302 response (Kostic et al., 2012). Furthermore, some studies have indicated that the genus 303 Fusobacterium was closely associated with obesity-related colorectal neoplasms (Amitay et al., 304 2017; McCoy et al., 2013), which may be one of the mechanisms that obese people are prone to 305 tumors. Thus, we inferred that these genera are strongly associated with obesity and may be 306 potential pathogens.

Consistent with previous studies of children with ASD (Berding \& Donovan, 2018), we

308 found that adults with ASD had a higher abundance of Ruminococcus (2.44\% increase). Previous

309 study has shown that the genera Ruminococcus could produce butyrate and alleviates insulin 310 resistance, which was beneficial to control obesity (Gao et al., 2018). 
311 Senegalimassilia, belonging to family Coriobacteriaceae, together with Clostridium

$312 X I V a$, has been identified as a p-cresol-producing intestinal bacteria (Saito et al., 2018). p-Cresol

313 can inhibit dopamine beta-hydroxylase (Southan, DeWolf \& Kruse, 1990), an enzyme catalyzing

314 the hydroxylation of dopamine to norepinephrine, which functions as a neurotransmitter. p-

315 Cresol may modulate behavioral abnormalities and autism severity, and high levels of p-cresol

316 are often observed in children with ASD (Persico \& Napolioni, 2013).

317 As a major genus in both groups, Blautia was significantly decreased (2.2\% decrease) in

318 obese group. Blautia plays an important role in nutrient assimilation, gut maturation, and

319 mucosal serotonin synthesis in the gut which accelerates gastrointestinal motility (Golubeva et

320 al., 2017; Liu et al., 2019). Agreement with our results, a previous study has indicated the genus

321 Blautia was decreased in obese adults, which was inversely association with visceral fat

322 accumulation (Ozato et al., 2019). So it may be a potential a potentially beneficial genus for 323 obese patients.

324 In our study, we also observed that the major genera Butyricicoccus, Clostridium IV, 325 Parasutterella, Parabacteroides, and Roseburia were decreased in obese group (Table 4). 326 Interestingly, recent study has shown that these genera were negatively associated with host's 327 BMI and lipid levels (Zeng et al., 2019). Among these genera, Butyricicoccus (Takada et al., 328 2016), Clostridium IV (Moens \& De Vuyst, 2017) and Roseburia (Kasahara et al., 2018) can 329 produce butyrate which has anti-inflammatory functions, thus being beneficial to anti-obesity. 330 Moreover, an animal study has shown that Parabacteroides is beneficial for reducing host 331 weight and hyperglycemia (Wang et al., 2019). Therefore, above-mentioned bacteria may be 332 beneficial in controlling obesity.

333 At the species level, two Blautia species (B. wexlerae and B. faeces) and R. faecis were 334 significantly increased in adults with ASD (Table 6). Kasai et al. observed that B. wexlerae was 335 significantly reduced in obese group compared to non-obese (Kasai et al., 2015). B. wexlerae is 336 also a major acetate producer (Jang et al., 2019). When the abundance of $B$. wexlerae decreased, 
337 the production of acetate and butyric acid was also decreased (Jang et al., 2019; Vital et al., 338 2018). However, animal experiment has shown that butyrate can improve insulin resistance and 339 reduce fat accumulation (Khan \& Jena, 2016). Therefore, this may be one of the mechanisms of 340 B. wexlerae anti-obesity.

341 Additionally, we also observed that the Dialister succinatiphilus, Megamonas funiformis 342 and Fusobacterium mortiferum were enriched in obese group. M. funiformis and F. mortiferum 343 belong to Gram-negative bacteria (Sakon et al., 2008), which their cell walls contain more

344 lipopolysaccharides that can induce or aggravate the host to produce inflammatory response and 345 insulin resistance, thus involving in the occurrence and development of obesity (Muscogiuri et 346 al., 2019). Additionally, study indicated that D. succinatiphilus is a succinate-utilizing bacteria

347 (Nakayama et al., 2017). Morotomi et al. have shown that succinate can stimulate the growth and 348 reproduction of $D$. succinatiphilus, while producing a large amount of propionate (Morotomi et 349 al., 2008). Interestingly, Ren et al. have found that circulating succinate concentrations was 350 increased in patients with obesity or type 2 diabetes (Ren et al., 2019). Moreover, Ceperuelo-

351 Mallafré et al. have indicated that succinate concentrations was significantly decreased in serum 352 of patients with diabetes after bariatric surgery, and considered baseline succinate levels to have 353 an independent predictive effect on diabetic remission (Ceperuelo-Mallafre et al., 2019). 354 Therefore, we speculated that the relative abundance of $D$. succinatiphilus may be as a biomarker 355 for predicting obesity.

356 Consistent with our findings, Serena et al. observed a lower abundance of 357 Phascolarctobacterium spp. in obese individuals than in non-obese people (Serena et al., 2018), 358 which is known as succinate-utilizing bacterium that may be affecting the energy metabolism of 359 the host by participating in the metabolism of succinate, thus reducing the occurrence of obesity 360 in the host. Interestingly, Phascolarctobacterium succinatuten, an asaccharolytic bacteria 361 distributed broadly in the gastrointestinal tract, can utilize succinate generated by other intestinal

362 bacterial species to produce propionate (Watanabe, Nagai \& Morotomi, 2012), which can cross 
363 the blood - brain barrier and act as a neurotoxin to elicit ASD-like behavior (Berding \&

364 Donovan, 2016).

365 In addition, the most abundant species in both groups was Prevotella copri, which 366 showed no significant difference between the ASD and obese groups (Table 6). Some studies

367 have shown that P. copri were involved in occurrence of obesity (Stanislawski et al., 2019)

368 through promoting the biosynthesis of branched-chain amino acids to induce insulin resistance

369 (Pedersen et al., 2016) and stimulating the secretion of inflammatory factors to trigger or 370 aggravate the host's inflammatory response (Larsen, 2017). Therefore, $P$. copri is associated with 371 both ASD and obesity, which may be a common-owned biomarker of ASD and obesity.

372 This is the first study to compare the microbial composition between ASD patients and 373 obesity adults. Nevertheless, the current study has several limitations. First, the sample size of 374 the study was relatively small, and both underweight $(n=5)$ and obese $(n=2)$ adult ASD were

375 included in the 21 patients. The small sample size limited our further grouping and comparison

376 between obese ASD and obesity. Indeed, we performed a comparison analysis between normal

377 weight ASDs $(n=14)$ and obesity, and most of the significant changes was similar as observed in 378 the comparison between all ASDs $(n=21)$ and obesity. However, the conclusion of this study is somewhat weakened, and more ASD adults including obese ASD will be recruited in subsequent studies. Second, all the DNA were extracted using QIAamp DNA stool mini kit, which had no

381 bead-beating step and was hard to lyse Gram-positive bacteria (Albertsen et al., 2015; Guo \&

382 Zhang, 2013). This might cause gram-positive bacteria to be underrepresented. Though it is not

383 critical to the conclusions since all the samples were processed similarly, a kit with bead-beating 384 step is preferred. Third, the diets of obese group were not uniform. Since the type of diet has a 
385 great influence on the gut microbiota, the dietary data should be collected and analyzed in further

386 studies.

387

388 Conclusions

389 In the present study, 42 fecal samples were collected from 21 adult patients with ASD and 21

390 obese adults. The gut microbiota composition was analyzed and compared to existing reports of

391 children with ASD or obesity. We found that the microbiota in adults with ASD exhibited higher

392 biodiversity than that of obese controls, with one phylum, seven families, 20 genera, and nine

393 species showing significant differences between the two groups. The two genera (Megamonas

394 and Fusobacterium) were significantly enriched in obese group. The propionate-producing

395 species $P$. succinatuten increased in adults with ASD. The species D. succinatiphilus may be as a

396 biomarker for predicting obesity, as well as P. copri may be a common-owned biomarker of

397 ASD and obesity. Furthermore, we observed that the unique intestinal microbiota is strongly

398 related to the occurrence and development of ASD or obesity, making the microbiota a potential

399 treatment target for patients with ASD or obese patients. More importantly, compared to

400 previous reports, we observed some conflicting results because of the different ages and obesity

401 status of the patients with ASD, which should be examined in further studies.

402

403 Acknowledgements

404 We would like to acknowledge all the participants and their families who kindly took part in this 405 research.

406

407 Funding

408 This work was supported by the Development Fund for Shanghai Talents [Grant number 409 201567]. 


\section{Disclosure Statement}

412 The authors declare that they have no competing interests.

413

\section{Authors' contributions}

415 Huajun Zheng and Quan Li designed the project. Sample collection was performed by Qiang 416 Zhang. DNA extraction and sequencing was performed by Min Guo and Mengmeng Duan.

417 Bioinformatics analysis was performed by Qiang Zhang and Rong Zou. The first draft of the 418 manuscript was written by Qiang Zhang and Huajun Zheng, and all authors commented on 419 previous versions of the manuscript. All authors read and approved the final manuscript.

\section{References}

422

423

424

425

426

427

428

429

430

431

432

433

434

435

436

437

438

439

440

441

442

443

Albertsen M, Karst SM, Ziegler AS, Kirkegaard RH, and Nielsen PH. 2015. Back to Basics--The Influence of DNA Extraction and Primer Choice on Phylogenetic Analysis of Activated Sludge Communities. PLoS One 10(7):e0132783. doi: 10.1371/journal.pone.0132783.

American Psychiatric Association. 2013. Diagnostic and Statistical Manual of Mental Disorders (DSM 5): Arlington: American Psychiatric Association Publishing.

Amitay EL, Werner S, Vital M, Pieper DH, Hofler D, Gierse IJ, Butt J, Balavarca Y, Cuk K, and Brenner H. 2017. Fusobacterium and colorectal cancer: causal factor or passenger? Results from a large colorectal cancer screening study. Carcinogenesis 38(8):781-788. doi: 10.1093/carcin/bgx053.

Andoh A, Nishida A, Takahashi K, Inatomi O, Imaeda H, Bamba S, Kito K, Sugimoto M, and Kobayashi T. 2016. Comparison of the gut microbial community between obese and lean peoples using $16 \mathrm{~S}$ gene sequencing in a Japanese population. J Clin Biochem Nutr 59(1):65-70. doi: 10.3164/jcbn.15-152.

Berding K, and Donovan SM. 2016. Microbiome and nutrition in autism spectrum disorder: current knowledge and research needs. Nutr Rev 74(12):723-736. doi: 10.1093/nutrit/nuw048.

Berding K, and Donovan SM. 2018. Diet Can Impact Microbiota Composition in Children With Autism Spectrum Disorder. Front Neurosci 12:515. doi: 10.3389/fnins.2018.00515.

Biddle A, Stewart L, Blanchard J, and Leschine S. 2013. Untangling the Genetic Basis of Fibrolytic Specialization by Lachnospiraceae and Ruminococcaceae in Diverse Gut Communities. Diversity 5(3):627-640. doi: $10.3390 / \mathrm{d} 5030627$.

Bray GA, Heisel WE, Afshin A, Jensen MD, Dietz WH, Long M, Kushner RF, Daniels SR, Wadden TA, Tsai AG, Hu FB, Jakicic JM, Ryan DH, Wolfe BM, and Inge TH. 2018. The Science of Obesity Management: An Endocrine Society Scientific Statement. Endocr Rev 39(2):79-132. doi: 10.1210/er.2017-00253.

Ceperuelo-Mallafre V, Llaurado G, Keiran N, Benaiges E, Astiarraga B, Martinez L, Pellitero S, Gonzalez-Clemente

Peer) reviewing PDF | (2020:08:52367:2:0:NEW 16 Jan 2021) 
444

445

446

447

448

449

450

451

452

453

454

455

456

457

458

459

460

461

462

463

464

465

466

467

468

469

470

471

472

473

474

475

476

477

478

479

480

481

482

JM, Rodriguez A, Fernandez-Real JM, Lecube A, Megia A, Vilarrasa N, Vendrell J, and Fernandez-Veledo S. 2019. Preoperative Circulating Succinate Levels as a Biomarker for Diabetes Remission After Bariatric Surgery. Diabetes Care 42(10):1956-1965. doi: 10.2337/dc19-0114.

Chiu CM, Huang WC, Weng SL, Tseng HC, Liang C, Wang WC, Yang T, Yang TL, Weng CT, Chang TH, and Huang HD. 2014. Systematic analysis of the association between gut flora and obesity through high-throughput sequencing and bioinformatics approaches. Biomed Res Int 2014:906168. doi: 10.1155/2014/906168.

Cole JR, Wang Q, Cardenas E, Fish J, Chai B, Farris RJ, Kulam-Syed-Mohideen AS, McGarrell DM, Marsh T, Garrity GM, and Tiedje JM. 2009. The Ribosomal Database Project: improved alignments and new tools for rRNA analysis. Nucleic Acids Res 37(Database issue):D141-145. doi: 10.1093/nar/gkn879.

Coretti L, Paparo L, Riccio MP, Amato F, Cuomo M, Natale A, Borrelli L, Corrado G, Comegna M, Buommino E, Castaldo G, Bravaccio C, Chiariotti L, Berni Canani R, and Lembo F. 2018. Gut Microbiota Features in Young Children With Autism Spectrum Disorders. Front Microbiol 9:3146. doi: 10.3389/fmicb.2018.03146.

De Angelis M, Piccolo M, Vannini L, Siragusa S, De Giacomo A, Serrazzanetti DI, Cristofori F, Guerzoni ME, Gobbetti M, and Francavilla R. 2013. Fecal microbiota and metabolome of children with autism and pervasive developmental disorder not otherwise specified. PLoS One 8(10):e76993. doi: 10.1371/journal.pone.0076993.

Dhaliwal KK, Orsso CE, Richard C, Haqq AM, and Zwaigenbaum L. 2019. Risk Factors for Unhealthy Weight Gain and Obesity among Children with Autism Spectrum Disorder. Int J Mol Sci 20(13). doi: 10.3390/ijms20133285.

Fattorusso A, Di Genova L, Dell'Isola GB, Mencaroni E, and Esposito S. 2019. Autism Spectrum Disorders and the Gut Microbiota. Nutrients 11(3). doi: 10.3390/nu11030521.

Fetissov SO. 2017. Role of the gut microbiota in host appetite control: bacterial growth to animal feeding behaviour. Nat Rev Endocrinol 13(1):11-25. doi: 10.1038/nrendo.2016.150.

Gao R, Zhu C, Li H, Yin M, Pan C, Huang L, Kong C, Wang X, Zhang Y, Qu S, and Qin H. 2018. Dysbiosis Signatures of Gut Microbiota Along the Sequence from Healthy, Young Patients to Those with Overweight and Obesity. Obesity (Silver Spring) 26(2):351-361. doi: 10.1002/oby.22088.

Golubeva AV, Joyce SA, Moloney G, Burokas A, Sherwin E, Arboleya S, Flynn I, Khochanskiy D, Moya-Perez A, Peterson V, Rea K, Murphy K, Makarova O, Buravkov S, Hyland NP, Stanton C, Clarke G, Gahan CGM, Dinan TG, and Cryan JF. 2017. Microbiota-related Changes in Bile Acid \& Tryptophan Metabolism are Associated with Gastrointestinal Dysfunction in a Mouse Model of Autism. EBioMedicine 24:166-178. doi: 10.1016/j.ebiom.2017.09.020.

Guo F, and Zhang T. 2013. Biases during DNA extraction of activated sludge samples revealed by high throughput sequencing. Appl Microbiol Biotechnol 97(10):4607-4616. doi: 10.1007/s00253-012-4244-4.

Healy S, Aigner CJ, and Haegele JA. 2019. Prevalence of overweight and obesity among US youth with autism spectrum disorder. Autism 23(4):1046-1050. doi: 10.1177/1362361318791817.

Hill AP, Zuckerman KE, and Fombonne E. 2015. Obesity and Autism. Pediatrics 136(6):1051-1061. doi: 10.1542/peds.2015-1437.

Huse SM, Huber JA, Morrison HG, Sogin ML, and Welch DM. 2007. Accuracy and quality of massively parallel DNA pyrosequencing. Genome Biol 8(7):R143. doi: 10.1186/gb-2007-8-7-r143.

Jang LG, Choi G, Kim SW, Kim BY, Lee S, and Park H. 2019. The combination of sport and sport-specific diet is

Peer] reviewing PDF | (2020:08:52367:2:0:NEW 16 Jan 2021) 
483

484

485

486

487

488

489

490

491

492

493

494

495

496

497

498

499

500

501

502

503

504

505

506

507

508

509

510

511

512

513

514

515

516

517

518

519

520

521 associated with characteristics of gut microbiota: an observational study. J Int Soc Sports Nutr 16(1):21. doi: 10.1186/s12970-019-0290-y.

Jensen MD, Ryan DH, Apovian CM, Ard JD, Comuzzie AG, Donato KA, Hu FB, Hubbard VS, Jakicic JM, Kushner RF, Loria CM, Millen BE, Nonas CA, Pi-Sunyer FX, Stevens J, Stevens VJ, Wadden TA, Wolfe BM, Yanovski SZ, Jordan HS, Kendall KA, Lux L, Mentor-Marcel R, Morgan LC, Trisolini MG, Wnek J, Anderson JL, Halperin JL, Albert NM, Bozkurt B, Brindis RG, Curtis LH, DeMets D, Hochman JS, Kovacs RJ, Ohman EM, Pressler SJ, Sellke FW, Shen WK, Smith SC, Jr., Tomaselli GF, American College of Cardiology/American Heart Association Task Force on Practice G, and Obesity S. 2014. 2013 AHA/ACC/TOS guideline for the management of overweight and obesity in adults: a report of the American College of Cardiology/American Heart Association Task Force on Practice Guidelines and The Obesity Society. Circulation 129(25 Suppl 2):S102-138. doi: 10.1161/01.cir.0000437739.71477.ee.

Kasahara K, Krautkramer KA, Org E, Romano KA, Kerby RL, Vivas El, Mehrabian M, Denu JM, Backhed F, Lusis AJ, and Rey FE. 2018. Interactions between Roseburia intestinalis and diet modulate atherogenesis in a murine model. Nat Microbiol 3(12):1461-1471. doi: 10.1038/s41564-018-0272-x.

Kasai C, Sugimoto K, Moritani I, Tanaka J, Oya Y, Inoue H, Tameda M, Shiraki K, Ito M, Takei Y, and Takase K. 2015. Comparison of the gut microbiota composition between obese and non-obese individuals in a Japanese population, as analyzed by terminal restriction fragment length polymorphism and next-generation sequencing. BMC Gastroenterol 15:100. doi: 10.1186/s12876-015-0330-2.

Khan MJ, Gerasimidis K, Edwards CA, and Shaikh MG. 2016. Role of Gut Microbiota in the Aetiology of Obesity: Proposed Mechanisms and Review of the Literature. J Obes 2016:7353642. doi: 10.1155/2016/7353642.

Khan S, and Jena G. 2016. Sodium butyrate reduces insulin-resistance, fat accumulation and dyslipidemia in type-2 diabetic rat: A comparative study with metformin. Chem Biol Interact 254:124-134. doi: 10.1016/j.cbi.2016.06.007.

Kieler IN, Shamzir Kamal S, Vitger AD, Nielsen DS, Lauridsen C, and Bjornvad CR. 2017. Gut microbiota composition may relate to weight loss rate in obese pet dogs. Vet Med Sci 3(4):252-262. doi: 10.1002/vms3.80.

Koliada A, Syzenko G, Moseiko V, Budovska L, Puchkov K, Perederiy V, Gavalko Y, Dorofeyev A, Romanenko M, Tkach S, Sineok L, Lushchak O, and Vaiserman A. 2017. Association between body mass index and Firmicutes/Bacteroidetes ratio in an adult Ukrainian population. BMC Microbiol 17(1):120. doi: 10.1186/s12866-017-1027-1.

Kostic AD, Gevers D, Pedamallu CS, Michaud M, Duke F, Earl AM, Ojesina Al, Jung J, Bass AJ, Tabernero J, Baselga J, Liu C, Shivdasani RA, Ogino S, Birren BW, Huttenhower C, Garrett WS, and Meyerson M. 2012. Genomic analysis identifies association of Fusobacterium with colorectal carcinoma. Genome Res 22(2):292-298. doi: 10.1101/gr.126573.111.

Langille MG, Zaneveld J, Caporaso JG, McDonald D, Knights D, Reyes JA, Clemente JC, Burkepile DE, Vega Thurber RL, Knight R, Beiko RG, and Huttenhower C. 2013. Predictive functional profiling of microbial communities using 16S rRNA marker gene sequences. Nat Biotechnol 31(9):814-821. doi: 10.1038/nbt.2676.

Larsen JM. 2017. The immune response to Prevotella bacteria in chronic inflammatory disease. Immunology 151(4):363-374. doi: 10.1111/imm.12760.

Lewis K, Lutgendorff F, Phan V, Soderholm JD, Sherman PM, and McKay DM. 2010. Enhanced translocation of 
522

523

524

525

526

527

528

529

530

531

532

533

534

535

536

537

538

539

540

541

542

543

544

545

546

547

548

549

550

551

552

553

554

555

556

557

558

559

560 bacteria across metabolically stressed epithelia is reduced by butyrate. Inflamm Bowel Dis 16(7):11381148. doi: 10.1002/ibd.21177.

Liu F, Li J, Wu F, Zheng H, Peng Q, and Zhou H. 2019. Altered composition and function of intestinal microbiota in autism spectrum disorders: a systematic review. Transl Psychiatry 9(1):43. doi: 10.1038/s41398-019-03896.

Luna RA, Savidge TC, and Williams KC. 2016. The Brain-Gut-Microbiome Axis: What Role Does It Play in Autism Spectrum Disorder? Curr Dev Disord Rep 3(1):75-81. doi: 10.1007/s40474-016-0077-7.

Ma B, Liang J, Dai M, Wang J, Luo J, Zhang Z, and Jing J. 2019. Altered Gut Microbiota in Chinese Children With Autism Spectrum Disorders. Front Cell Infect Microbiol 9:40. doi: 10.3389/fcimb.2019.00040.

MacFabe DF. 2015. Enteric short-chain fatty acids: microbial messengers of metabolism, mitochondria, and mind: implications in autism spectrum disorders. Microb Ecol Health Dis 26:28177. doi: 10.3402/mehd.v26.28177.

Martin CR, Osadchiy V, Kalani A, and Mayer EA. 2018. The Brain-Gut-Microbiome Axis. Cell Mol Gastroenterol Hepatol 6(2):133-148. doi: 10.1016/j.jcmgh.2018.04.003.

Maya-Lucas O, Murugesan S, Nirmalkar K, Alcaraz LD, Hoyo-Vadillo C, Pizano-Zarate ML, and Garcia-Mena J. 2019. The gut microbiome of Mexican children affected by obesity. Anaerobe 55:11-23. doi: 10.1016/j.anaerobe.2018.10.009.

McCoy AN, Araujo-Perez F, Azcarate-Peril A, Yeh JJ, Sandler RS, and Keku TO. 2013. Fusobacterium is associated with colorectal adenomas. PLoS One 8(1):e53653. doi: 10.1371/journal.pone.0053653.

McCreath KJ, Espada S, Galvez BG, Benito M, de Molina A, Sepulveda P, and Cervera AM. 2015. Targeted disruption of the SUCNR1 metabolic receptor leads to dichotomous effects on obesity. Diabetes 64(4):1154-1167. doi: 10.2337/db14-0346.

Mitev K, and Taleski V. 2019. Association between the Gut Microbiota and Obesity. Open Access Maced J Med Sci 7(12):2050-2056. doi: 10.3889/oamjms.2019.586.

Moens F, and De Vuyst L. 2017. Inulin-type fructan degradation capacity of Clostridium cluster IV and XIVa butyrate-producing colon bacteria and their associated metabolic outcomes. Benef Microbes 8(3):473-490. doi: 10.3920/BM2016.0142.

Moreno-Indias I, Sanchez-Alcoholado L, Garcia-Fuentes E, Cardona F, Queipo-Ortuno MI, and Tinahones FJ. 2016. Insulin resistance is associated with specific gut microbiota in appendix samples from morbidly obese patients. Am J Transl Res 8(12):5672-5684. doi:

Morotomi M, Nagai F, Sakon H, and Tanaka R. 2008. Dialister succinatiphilus sp. nov. and Barnesiella intestinihominis sp. nov., isolated from human faeces. Int J Syst Evol Microbiol 58(Pt 12):2716-2720. doi: 10.1099/ijs.0.2008/000810-0.

Muscogiuri G, Cantone E, Cassarano S, Tuccinardi D, Barrea L, Savastano S, Colao A, on behalf of the Obesity Programs of nutrition ER, and Assessment g. 2019. Gut microbiota: a new path to treat obesity. Int J Obes Supp/ 9(1):10-19. doi: 10.1038/s41367-019-0011-7.

Nakayama J, Yamamoto A, Palermo-Conde LA, Higashi K, Sonomoto K, Tan J, and Lee YK. 2017. Impact of Westernized Diet on Gut Microbiota in Children on Leyte Island. Front Microbiol 8:197. doi: 10.3389/fmicb.2017.00197.

Peer) reviewing PDF | (2020:08:52367:2:0:NEW 16 Jan 2021) 
561 Ogden CL, Carroll MD, Kit BK, and Flegal KM. 2014. Prevalence of childhood and adult obesity in the United States,

562 2011-2012. JAMA 311(8):806-814. doi: 10.1001/jama.2014.732.

563 Ozato N, Saito S, Yamaguchi T, Katashima M, Tokuda I, Sawada K, Katsuragi Y, Kakuta M, Imoto S, Ihara K, and

$564 \quad$ Nakaji S. 2019. Blautia genus associated with visceral fat accumulation in adults 20-76 years of age. NPJ

565

566 Biofilms Microbiomes 5:28. doi: 10.1038/s41522-019-0101-x.

567

Parks DH, Tyson GW, Hugenholtz P, and Beiko RG. 2014. STAMP: statistical analysis of taxonomic and functional

568 profiles. Bioinformatics 30(21):3123-3124. doi: 10.1093/bioinformatics/btu494.

Pedersen HK, Gudmundsdottir V, Nielsen HB, Hyotylainen T, Nielsen T, Jensen BA, Forslund K, Hildebrand F, Prifti E,

569 Falony G, Le Chatelier E, Levenez F, Dore J, Mattila I, Plichta DR, Poho P, Hellgren LI, Arumugam M,

570

571 Sunagawa S, Vieira-Silva S, Jorgensen T, Holm JB, Trost K, Meta HITC, Kristiansen K, Brix S, Raes J, Wang J,

572 Hansen T, Bork P, Brunak S, Oresic M, Ehrlich SD, and Pedersen O. 2016. Human gut microbes impact host

573 serum metabolome and insulin sensitivity. Nature 535(7612):376-381. doi: 10.1038/nature18646.

Persico AM, and Napolioni V. 2013. Urinary p-cresol in autism spectrum disorder. Neurotoxicol Teratol 36:82-90. doi: 10.1016/j.ntt.2012.09.002.

575

Quast C, Pruesse E, Yilmaz P, Gerken J, Schweer T, Yarza P, Peplies J, and Glockner FO. 2013. The SILVA ribosomal

576

577

578

579

580

581

582

583

584

585

586

587

588

589 RNA gene database project: improved data processing and web-based tools. Nucleic Acids Res 41(Database issue):D590-596. doi: 10.1093/nar/gks1219.

Ren W, Xia Y, Chen S, Wu G, Bazer FW, Zhou B, Tan B, Zhu G, Deng J, and Yin Y. 2019. Glutamine Metabolism in Macrophages: A Novel Target for Obesity/Type 2 Diabetes. Adv Nutr 10(2):321-330. doi: 10.1093/advances/nmy084.

Saito Y, Sato T, Nomoto K, and Tsuji H. 2018. Identification of phenol- and p-cresol-producing intestinal bacteria by using media supplemented with tyrosine and its metabolites. FEMS Microbiol Ecol 94(9). doi: 10.1093/femsec/fiy125.

Sakon H, Nagai F, Morotomi M, and Tanaka R. 2008. Sutterella parvirubra sp. nov. and Megamonas funiformis sp. nov., isolated from human faeces. Int J Syst Evol Microbiol 58(Pt 4):970-975. doi: 10.1099/ijs.0.65456-0.

Schloss PD, Gevers D, and Westcott SL. 2011. Reducing the effects of PCR amplification and sequencing artifacts on 16S rRNA-based studies. PLoS One 6(12):e27310. doi: 10.1371/journal.pone.0027310.

Sedgewick F, Leppanen J, and Tchanturia K. 2020. Autistic adult outcomes on weight and body mass index: a largescale online study. Eat Weight Disord 25(3):795-801. doi: 10.1007/s40519-019-00695-8.

590

591

592

593

594

595

596

597

Serena C, Ceperuelo-Mallafre V, Keiran N, Queipo-Ortuno MI, Bernal R, Gomez-Huelgas R, Urpi-Sarda M, Sabater M, Perez-Brocal V, Andres-Lacueva C, Moya A, Tinahones FJ, Fernandez-Real JM, Vendrell J, and FernandezVeledo S. 2018. Elevated circulating levels of succinate in human obesity are linked to specific gut microbiota. ISME J 12(7):1642-1657. doi: 10.1038/s41396-018-0068-2.

Southan C, DeWolf WE, Jr., and Kruse LI. 1990. Inactivation of dopamine beta-hydroxylase by p-cresol: evidence for a second, minor site of covalent modification at tyrosine 357. Biochim Biophys Acta 1037(2):256-258. doi: 10.1016/0167-4838(90)90176-g.

Stanislawski MA, Dabelea D, Lange LA, Wagner BD, and Lozupone CA. 2019. Gut microbiota phenotypes of obesity. NPJ Biofilms Microbiomes 5:18. doi: 10.1038/s41522-019-0091-8.

Takada T, Watanabe K, Makino H, and Kushiro A. 2016. Reclassification of Eubacterium desmolans as

Peer] reviewing PDF | (2020:08:52367:2:0:NEW 16 Jan 2021) 
600

601

602

603

604

605

606

607

608

609

610

611

612

613

614

615

616

617

618

619

620

621

622

623

624

625

626

627

628

629

630

631

632

633

634

\section{Figure Legends} Switzerland. 019-49462-w. 018-32219-2.

Butyricicoccus desmolans comb. nov., and description of Butyricicoccus faecihominis sp. nov., a butyrateproducing bacterium from human faeces. Int J Syst Evol Microbiol 66(10):4125-4131. doi: 10.1099/ijsem.0.001323.

Taylor R. 1990. Interpretation of the Correlation Coefficient: A Basic Review. ournal of Diagnostic Medical Sonography 6(1):35-39. doi: 10.1177/875647939000600106.

Torres-Fuentes C, Schellekens H, Dinan TG, and Cryan JF. 2017. The microbiota-gut-brain axis in obesity. Lancet Gastroenterol Hepatol 2(10):747-756. doi: 10.1016/S2468-1253(17)30147-4.

van Sadelhoff JHJ, Perez Pardo P, Wu J, Garssen J, van Bergenhenegouwen J, Hogenkamp A, Hartog A, and Kraneveld AD. 2019. The Gut-Immune-Brain Axis in Autism Spectrum Disorders; A Focus on Amino Acids. Front Endocrinol (Lausanne) 10:247. doi: 10.3389/fendo.2019.00247.

Vital M, Howe A, Bergeron N, Krauss RM, Jansson JK, and Tiedje JM. 2018. Metagenomic Insights into the Degradation of Resistant Starch by Human Gut Microbiota. Appl Environ Microbiol 84(23). doi: 10.1128/AEM.01562-18.

Wang K, Liao M, Zhou N, Bao L, Ma K, Zheng Z, Wang Y, Liu C, Wang W, Wang J, Liu SJ, and Liu H. 2019. Parabacteroides distasonis Alleviates Obesity and Metabolic Dysfunctions via Production of Succinate and Secondary Bile Acids. Cell Rep 26(1):222-235 e225. doi: 10.1016/j.celrep.2018.12.028.

Watanabe Y, Nagai F, and Morotomi M. 2012. Characterization of Phascolarctobacterium succinatutens sp. nov., an asaccharolytic, succinate-utilizing bacterium isolated from human feces. Appl Environ Microbiol 78(2):511-518. doi: 10.1128/AEM.06035-11.

World Health Organization. 1993. The ICD-10 Classification of Mental and Behavioural Disorders. Genève,

Zeng Q, Li D, He Y, Li Y, Yang Z, Zhao X, Liu Y, Wang Y, Sun J, Feng X, Wang F, Chen J, Zheng Y, Yang Y, Sun X, Xu X, Wang D, Kenney T, Jiang Y, Gu H, Li Y, Zhou K, Li S, and Dai W. 2019. Discrepant gut microbiota markers for the classification of obesity-related metabolic abnormalities. Sci Rep 9(1):13424. doi: 10.1038/s41598-

Zhang M, Ma W, Zhang J, He Y, and Wang J. 2018. Analysis of gut microbiota profiles and microbe-disease associations in children with autism spectrum disorders in China. Sci Rep 8(1):13981. doi: 10.1038/s41598-

Zheng Z, Zhang L, Li S, Zhao F, Wang Y, Huang L, Huang J, Zou R, Qu Y, and Mu D. 2017. Association among obesity, overweight and autism spectrum disorder: a systematic review and meta-analysis. Sci Rep 7(1):11697. doi: 10.1038/s41598-017-12003-4.

Zou R, Xu F, Wang Y, Duan M, Guo M, Zhang Q, Zhao H, and Zheng H. 2020. Changes in the Gut Microbiota of Children with Autism Spectrum Disorder. Autism Res. doi: 10.1002/aur.2358.

Peer) reviewing PDF | (2020:08:52367:2:0:NEW 16 Jan 2021) 
636 Figure 1. Principal component analysis (PCA) calculated by weighted UniFrac distances.

637 (A) The 21 ASD adults were divided into three groups. (B) All the 21 ASD adults were 638 taken as one group. Points representing samples were colored according to groups.

639

640 Figure 2. Comparison of bacterial richness, evenness, and diversity between ASD and

641 obesity groups. (A) ACE index, (B) Chao index, (C) Shannon evenness index, and (D)

642 Shannon diversity index were compared by Student $t$-test.

643

644 Figure 3. Comparison of families between ASD and obesity microbiota. The p-values was 645 calculated based on two-sided Welch's t-test.

646

647 Figure 4. Comparison of genera between ASD and obesity microbiota. The p-values was 648 calculated based on two-sided Welch's t-test.

649

650

Figure 5. Difference in functional pathway prediction using PICRUSt for ASD and obesity gut microbiota. A: Metabolism; B: Environmental information processing. The p-values was calculated based on two-sided Welch's t-test.

653

654 Figure 6. Correlations between species calculated using Spearman correlation algorithm.

655 The light yellow part is the correlation species of ASD microbiota, and the light blue part is the 
656 shared correlation species by ASD and obesity microbiota, while the other part was the

657 correlation species of obesity microbiota. The pie charts show relative species proportions in 658 ASD (yellow) and obesity groups (blue), and the circle size represents the read number. Line 659 color: Green (positive relationship) and grey (negative relationship).

660

661 Tables

662 Table 1. Diversity evaluation of two groups microbiota.

663

664 Table 2. Significantly different phyla of gut microbiota between ASD and obesity.

665

666 Table 3. Major abundant and significantly different families in ASD and obesity gut 667 microbiota.

668

669 Table 4. Major abundant and significantly different genera in difference gut microbiota.

671 Table 5. AMOVA analysis result between different groups based on microbiota 672 composition.

673

674 Table 6. Significantly different species of gut microbiota between ASD and obesity.

675

676 Table 7. Function prediction using PICRUSt based on 16S rRNA gene copy numbers.

677

678 Table 8. Correlations of species calculated using Spearman algorithm.

679 
680 Table 9. Significantly different taxa between normal weight ASD and Obesity in addition 681 to all ASD and Obesity. 
Figure 1

Principal component analysis (PCA) calculated by weighted UniFrac distances.

(A) The 21 ASD adults were divided into three groups. (B) All the 21 ASD adults were taken as one group. Points representing samples were colored according to groups.

A.

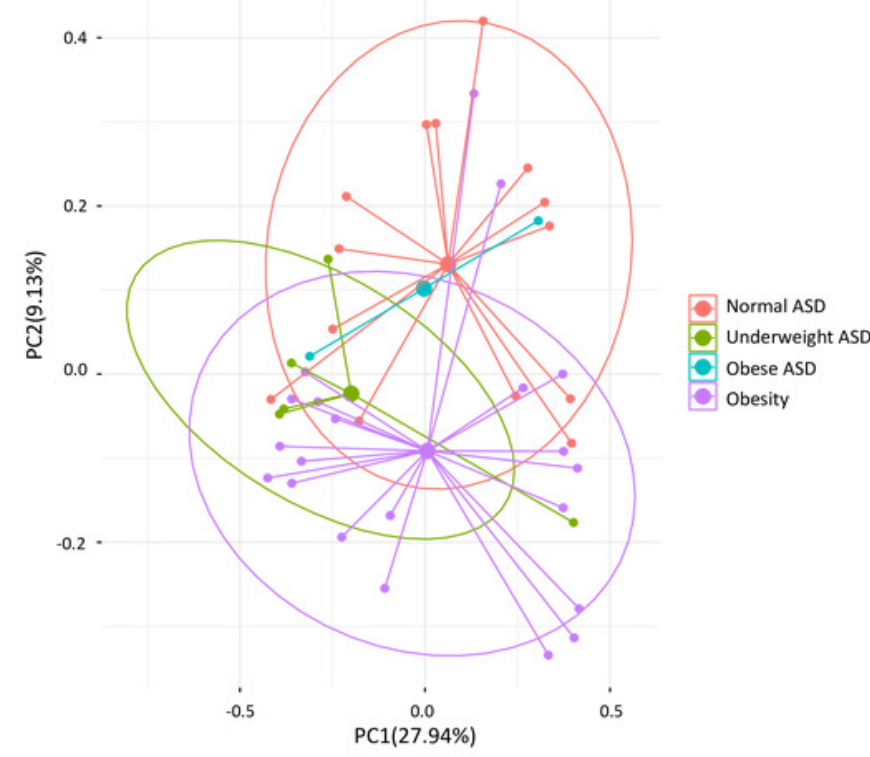

B.

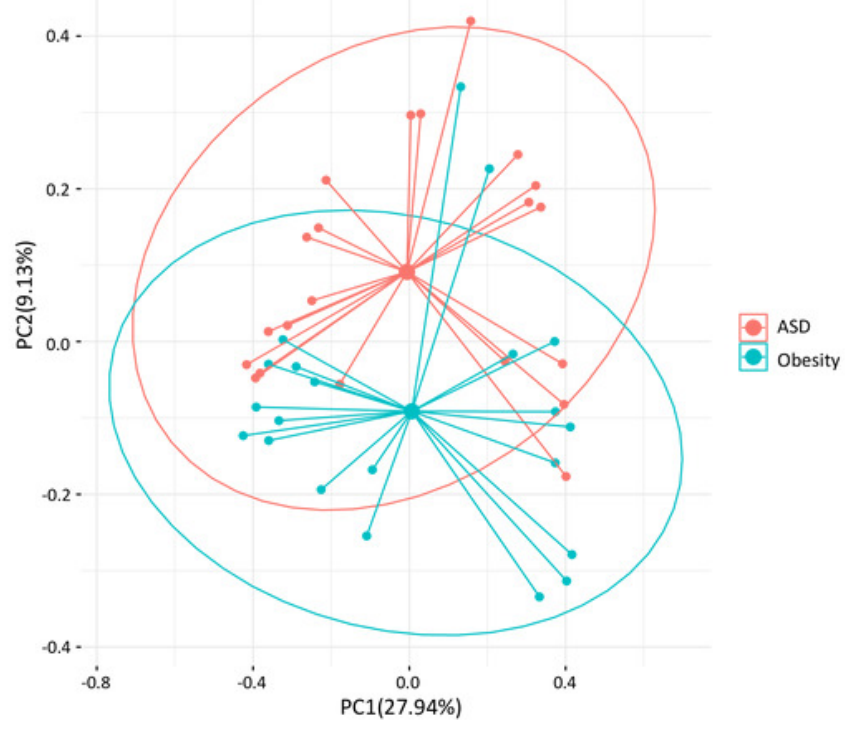


Figure 2

Comparison of bacterial richness, evenness, and diversity between ASD and obesity groups.

(A) ACE index, (B) Chao index, (C) Shannon evenness index, and (D) Shannon diversity index were compared by Student $t$-test.

A.

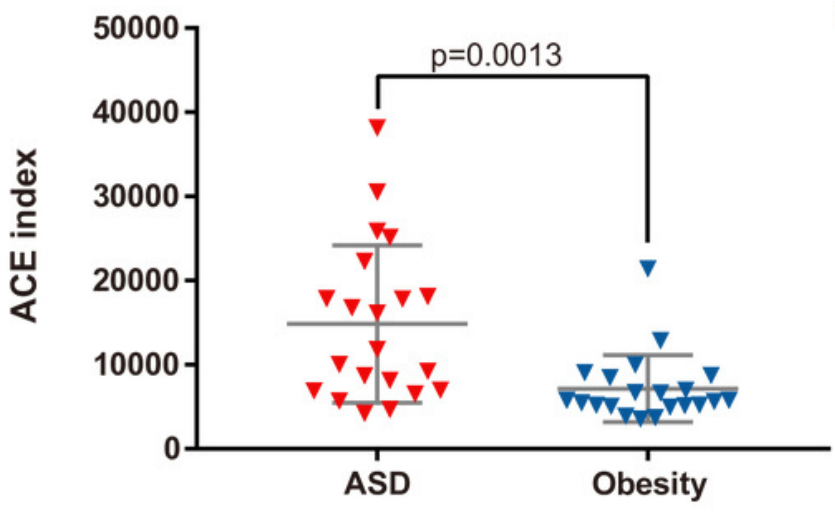

C.

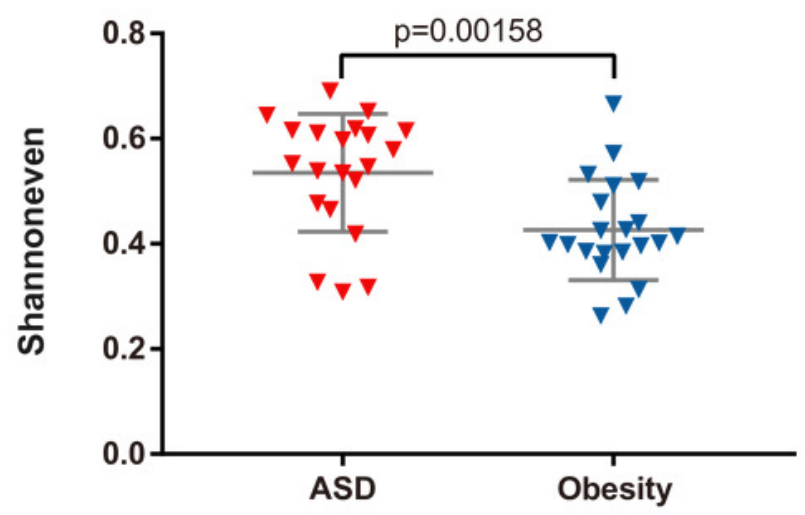

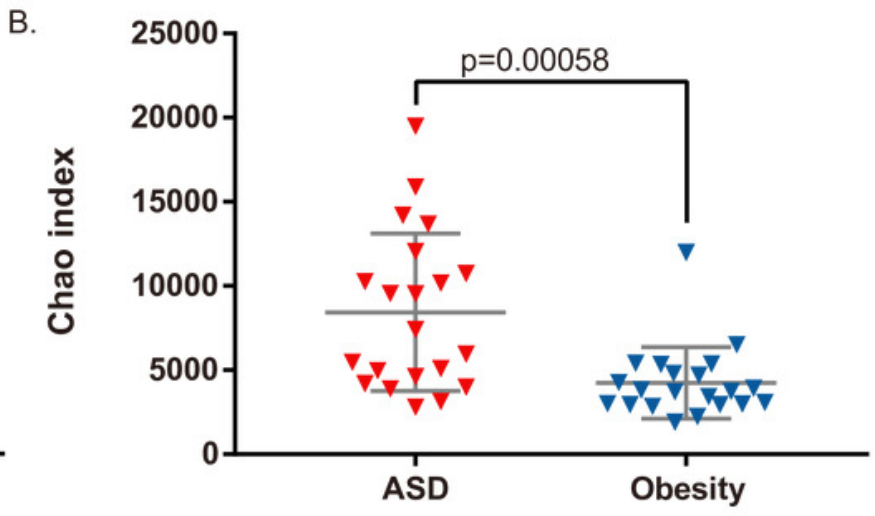

D.

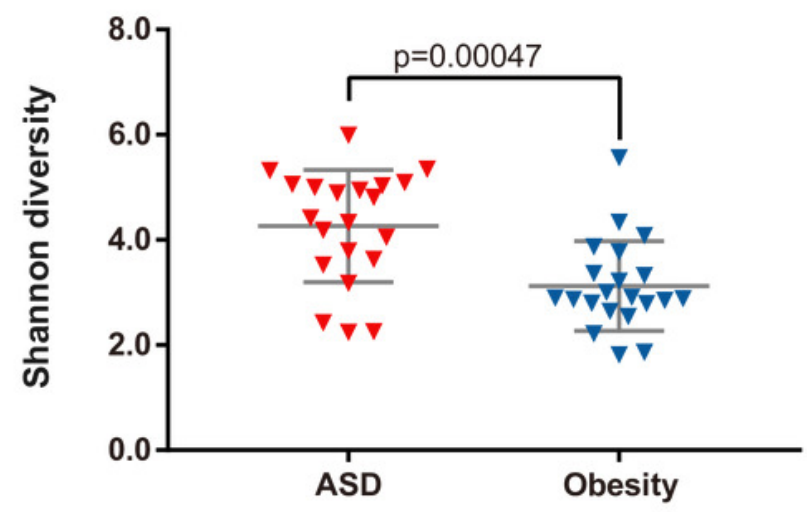


Figure 3

Comparison of families between ASD and obesity microbiota.

The p-values was calculated based on two-sided Welch's t-test.

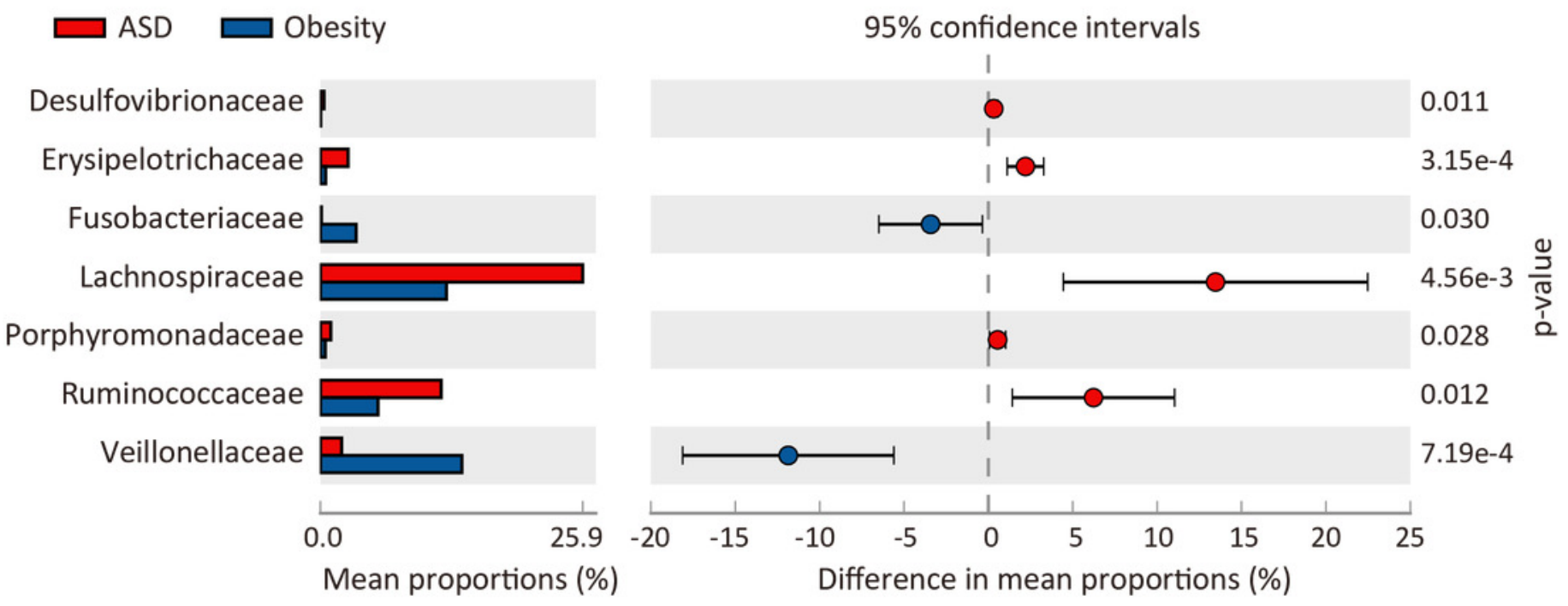


Figure 4

Comparison of genera between ASD and obesity microbiota.

The p-values was calculated based on two-sided Welch's t-test.

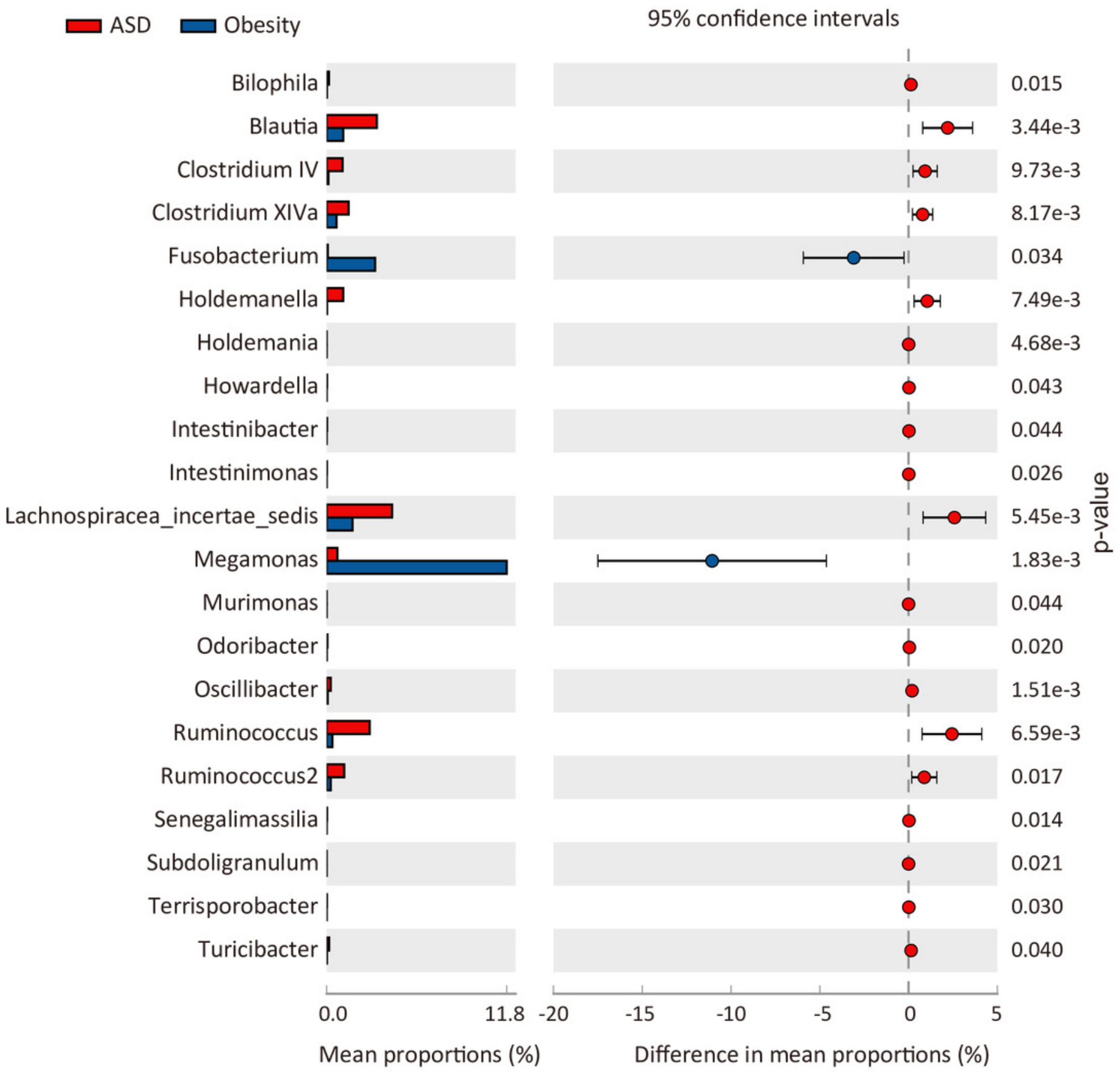


Figure 5

Difference in functional pathway prediction using PICRUSt for ASD and obesity gut microbiota.

A: Metabolism; B: Environmental information processing. The $p$-values was calculated based on two-sided Welch's t-test.

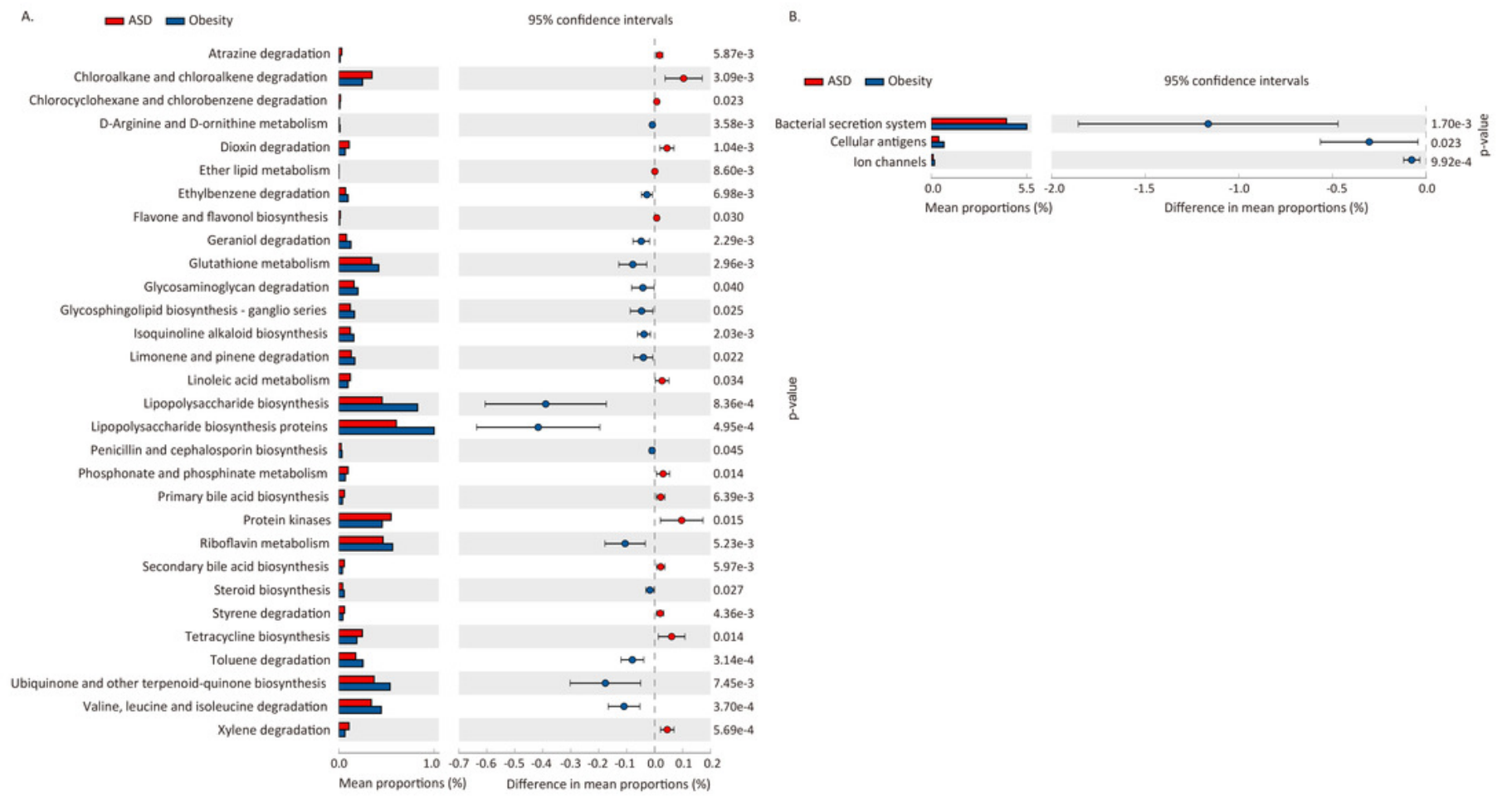




\section{Figure 6}

Correlations between species calculated using Spearman correlation algorithm.

The light yellow part is the correlation species of ASD microbiota, and the light blue part is the shared correlation species by ASD and obesity microbiota, while the other part was the correlation species of obesity microbiota. The pie charts show relative species proportions in ASD (yellow) and obesity groups (blue), and the circle size represents the read number. Line color: Green (positive relationship) and grey (negative relationship).

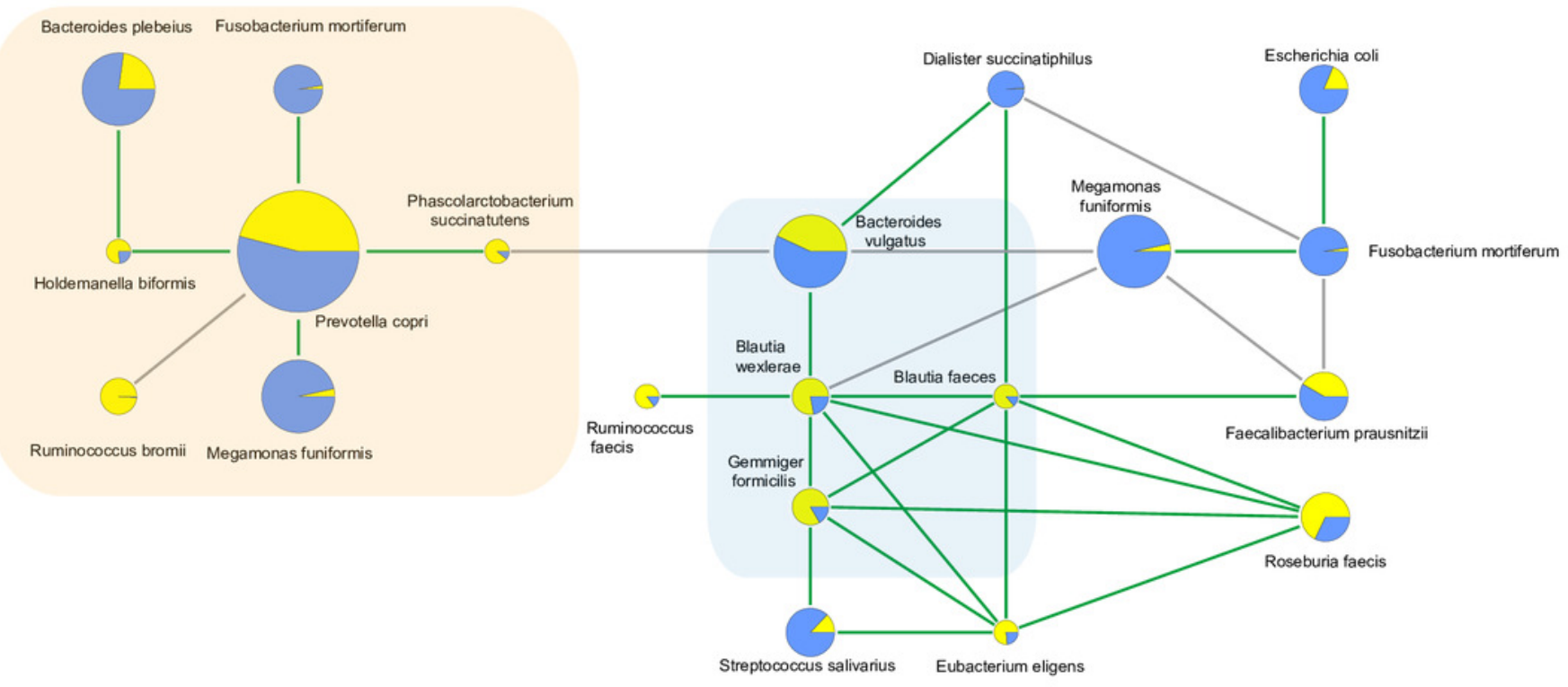




\section{Table 1 (on next page)}

Diversity evaluation of two groups microbiota. 
1 Table 1. Diversity evaluation of two groups microbiota.

\begin{tabular}{lcccccccc}
\hline \multirow{2}{*}{ Group } & \multirow{2}{*}{ Sample } & \multirow{2}{*}{ OTUs } & \multirow{2}{*}{ Coverage } & \multicolumn{2}{c}{ Richness } & \multicolumn{2}{c}{ Evenness } & \multicolumn{2}{c}{ Diversity } \\
\cline { 6 - 9 } & & & & Chao & ACE & simpsoneven & Shannon & Simpson \\
\hline ASD & 21 & 10511 & 0.999013 & 10837.95 & 10781.76 & 0.001651 & 5.223554 & 0.057639 \\
Normal weightt ASD & 14 & 9444 & 0.997592 & 10027.12 & 10070.15 & 0.00335 & 5.485606 & 0.031612 \\
Obesity & 21 & 7530 & 0.998214 & 8544.02 & 8437.36 & 0.001693 & 4.338984 & 0.078443 \\
\hline
\end{tabular}

2 


\section{Table 2 (on next page)}

Significantly different phyla of gut microbiota between ASD and obesity. 
1 Table 2. Significantly different phyla of gut microbiota between ASD and obesity.

\begin{tabular}{|c|c|c|c|c|c|c|c|c|}
\hline phylum & $\begin{array}{l}\text { ASD: mean } \\
\text { rel. freq. }(\%)\end{array}$ & $\begin{array}{l}\text { ASD: std. } \\
\text { dev. (\%) }\end{array}$ & $\begin{array}{l}\text { Obesity: } \\
\text { mean rel. } \\
\text { freq. (\%) }\end{array}$ & $\begin{array}{l}\text { Obesity: std. } \\
\text { dev. (\%) }\end{array}$ & p-values & $\begin{array}{l}\text { Difference } \\
\text { between } \\
\text { means }\end{array}$ & $\begin{array}{l}95.0 \% \text { lower } \\
\text { CI }\end{array}$ & $\begin{array}{l}95.0 \% \text { upper } \\
\text { CI }\end{array}$ \\
\hline Firmicutes & 49.74 & 18.31 & 37.55 & 20.09 & $5.18 \mathrm{E}-02$ & 12.18 & -0.10 & 24.47 \\
\hline Actinobacteria & 2.68 & 5.62 & 0.56 & 0.70 & $1.10 \mathrm{E}-01$ & 2.11 & -0.52 & 4.75 \\
\hline Verrucomicrobia & 1.01 & 4.47 & 0.01 & 0.04 & $3.28 \mathrm{E}-01$ & 1.00 & -1.08 & 3.09 \\
\hline unclassified_Bacteria & 0.94 & 0.45 & 0.67 & 0.29 & $3.34 \mathrm{E}-02$ & 0.26 & 0.02 & 0.51 \\
\hline Synergistetes & 0.08 & 0.34 & 0.00 & 0.00 & $3.19 \mathrm{E}-01$ & 0.08 & -0.08 & 0.24 \\
\hline Tenericutes & 0.00 & 0.00 & 0.00 & 0.00 & $2.14 \mathrm{E}-01$ & 0.00 & 0.00 & 0.00 \\
\hline Planctomycetes & 0.00 & 0.00 & 0.00 & 0.00 & $3.29 \mathrm{E}-01$ & 0.00 & 0.00 & 0.00 \\
\hline Fibrobacteres & 0.00 & 0.00 & 0.00 & 0.00 & $3.29 \mathrm{E}-01$ & 0.00 & 0.00 & 0.00 \\
\hline Spirochaetes & 0.00 & 0.00 & 0.00 & 0.00 & $3.29 \mathrm{E}-01$ & 0.00 & 0.00 & 0.00 \\
\hline Ignavibacteriae & 0.00 & 0.00 & 0.00 & 0.00 & $3.29 \mathrm{E}-01$ & 0.00 & 0.00 & 0.00 \\
\hline Gemmatimonadetes & 0.00 & 0.00 & 0.00 & 0.00 & $3.29 \mathrm{E}-01$ & 0.00 & 0.00 & 0.00 \\
\hline Acidobacteria & 0.00 & 0.00 & 0.00 & 0.00 & $1.84 \mathrm{E}-01$ & 0.00 & 0.00 & 0.00 \\
\hline Chloroflexi & 0.00 & 0.00 & 0.00 & 0.01 & $3.29 \mathrm{E}-01$ & 0.00 & 0.00 & 0.00 \\
\hline Candidatus Saccharibacteria & 0.00 & 0.00 & 0.01 & 0.01 & $6.98 \mathrm{E}-02$ & 0.00 & -0.01 & 0.00 \\
\hline Lentisphaerae & 0.00 & 0.01 & 0.06 & 0.27 & $3.47 \mathrm{E}-01$ & -0.06 & -0.18 & 0.07 \\
\hline Elusimicrobia & 0.00 & 0.00 & 0.20 & 0.88 & $3.29 \mathrm{E}-01$ & -0.20 & -0.61 & 0.21 \\
\hline Proteobacteria & 1.88 & 1.76 & 3.97 & 6.68 & $1.89 \mathrm{E}-01$ & -2.09 & -5.29 & 1.10 \\
\hline Fusobacteria & 0.10 & 0.24 & 3.52 & 6.57 & $3.08 \mathrm{E}-02$ & -3.41 & -6.48 & -0.35 \\
\hline Bacteroidetes & 43.56 & 20.92 & 53.44 & 21.67 & $1.50 \mathrm{E}-01$ & -9.88 & -23.49 & 3.74 \\
\hline
\end{tabular}

2 


\section{Table 3 (on next page)}

Major abundant and significantly different families in ASD and obesity gut microbiota. 
1 Table 3. Major abundant and significantly different families in ASD and obesity gut microbiota.

\begin{tabular}{|c|c|c|c|c|}
\hline family & feature & ASD & Obesity & Enriched in \\
\hline Bifidobacteriaceae & major \& ubiquitous & $2.37 \%$ & $0.38 \%$ & \\
\hline Bacteroidaceae & major \& ubiquitous & $10.87 \%$ & $19.43 \%$ & \\
\hline Porphyromonadaceae & major \& difference & $1.01 \%$ & $0.47 \%$ & ASD \\
\hline Prevotellaceae & major \& ubiquitous & $29.09 \%$ & $32.62 \%$ & \\
\hline Rikenellaceae & major & $1.96 \%$ & $0.11 \%$ & \\
\hline Streptococcaceae & major & $0.47 \%$ & $2.42 \%$ & \\
\hline Lachnospiraceae & major \& difference & $25.89 \%$ & $12.42 \%$ & ASD \\
\hline Ruminococcaceae & major \& difference & $11.91 \%$ & $5.68 \%$ & ASD \\
\hline Erysipelotrichaceae & major \& difference & $2.71 \%$ & $0.52 \%$ & ASD \\
\hline Acidaminococcaceae & major & $1.47 \%$ & $0.83 \%$ & \\
\hline Veillonellaceae & major \& difference & $2.09 \%$ & $13.95 \%$ & Obesity \\
\hline Fusobacteriaceae & major \& difference & $0.09 \%$ & $3.51 \%$ & Obesity \\
\hline Sutterellaceae & major & $0.53 \%$ & $1.01 \%$ & \\
\hline Desulfovibrionaceae & difference & $0.35 \%$ & $0.03 \%$ & ASD \\
\hline Enterobacteriaceae & major \& ubiquitous & $0.68 \%$ & $2.65 \%$ & \\
\hline Verrucomicrobiaceae & major & $1.01 \%$ & $0.01 \%$ & $\square$ \\
\hline
\end{tabular}

2

3

4

5

6

7

8

9

10 


\section{Table 4 (on next page)}

Major abundant and significantly different genera in difference gut microbiota. 
1 Table 4. Major abundant and significantly different genera in difference gut microbiota.

\begin{tabular}{|c|c|c|c|c|}
\hline genus & feature & ASD & Obesity & Enriched in \\
\hline Lachnospiracea_incertae_sedis & major \& difference \& ubiquitous & $4.28 \%$ & $1.70 \%$ & ASD \\
\hline Ruminococcus & major \& difference & $2.81 \%$ & $0.37 \%$ & ASD \\
\hline Blautia & major \& difference \& ubiquitous & $3.28 \%$ & $1.08 \%$ & ASD \\
\hline Holdemanella & major \& difference & $1.08 \%$ & $0.03 \%$ & ASD \\
\hline Clostridium IV & major \& difference & $1.04 \%$ & $0.12 \%$ & ASD \\
\hline Ruminococcus 2 & major \& difference \& ubiquitous & $1.14 \%$ & $0.26 \%$ & ASD \\
\hline Clostridium XlVa & major \& difference \& ubiquitous & $1.44 \%$ & $0.65 \%$ & ASD \\
\hline Oscillibacter & major \& difference & $0.26 \%$ & $0.07 \%$ & ASD \\
\hline Turicibacter & major \& difference & $0.16 \%$ & $0.02 \%$ & ASD \\
\hline Bilophila & major \& difference & $0.15 \%$ & $0.02 \%$ & ASD \\
\hline Odoribacter & difference & $0.05 \%$ & $0.01 \%$ & ASD \\
\hline Howardella & difference & $0.03 \%$ & $0.00 \%$ & ASD \\
\hline Senegalimassilia & difference & $0.03 \%$ & $0.00 \%$ & ASD \\
\hline Intestinibacter & difference & $0.02 \%$ & $0.00 \%$ & ASD \\
\hline Terrisporobacter & difference & $0.02 \%$ & $0.01 \%$ & ASD \\
\hline Intestinimonas & difference & $0.02 \%$ & $0.00 \%$ & ASD \\
\hline Holdemania & difference & $0.01 \%$ & $0.00 \%$ & ASD \\
\hline Murimonas & difference & $0.00 \%$ & $0.00 \%$ & ASD \\
\hline Fusobacterium & major \& difference & $0.08 \%$ & $3.17 \%$ & Obesity \\
\hline Megamonas & major \& difference & $0.70 \%$ & $11.77 \%$ & Obesity \\
\hline Bifidobacterium & major & $2.12 \%$ & $0.33 \%$ & \\
\hline Collinsella & major & $0.21 \%$ & $0.16 \%$ & \\
\hline Bacteroides & major \& ubiquitous & $10.87 \%$ & $19.43 \%$ & \\
\hline Parabacteroides & major & $0.58 \%$ & $0.32 \%$ & \\
\hline Barnesiella & major & $0.15 \%$ & $0.05 \%$ & \\
\hline Prevotella & major & $27.82 \%$ & $30.21 \%$ & \\
\hline Paraprevotella & major & $0.16 \%$ & $0.06 \%$ & \\
\hline Alloprevotella & major & $0.94 \%$ & $2.24 \%$ & \\
\hline Alistipes & major & $1.95 \%$ & $0.11 \%$ & \\
\hline Elusimicrobium & major & $0.00 \%$ & $0.20 \%$ & \\
\hline Lactobacillus & major & $0.06 \%$ & $0.11 \%$ & \\
\hline Streptococcus & major \& ubiquitous & $0.47 \%$ & $2.42 \%$ & \\
\hline Clostridium sensu stricto & major & $0.62 \%$ & $0.36 \%$ & \\
\hline Dorea & major \& ubiquitous & $0.28 \%$ & $0.20 \%$ & \\
\hline Clostridium XlVb & major & $0.31 \%$ & $0.54 \%$ & \\
\hline Coprococcus & major & $1.23 \%$ & $0.42 \%$ & \\
\hline Roseburia & major & $5.40 \%$ & $2.95 \%$ & \\
\hline Anaerostipes & major & $0.12 \%$ & $0.19 \%$ & \\
\hline
\end{tabular}




\begin{tabular}{llll} 
Fusicatenibacter & major & $0.65 \%$ & $0.34 \%$ \\
Butyrivibrio & major & $0.17 \%$ & $0.00 \%$ \\
Romboutsia & major & $0.49 \%$ & $0.22 \%$ \\
Faecalibacterium & major & $2.49 \%$ & $3.42 \%$ \\
Butyricicoccus & major & $0.19 \%$ & $0.25 \%$ \\
Gemmiger & major & $1.53 \%$ & $0.85 \%$ \\
Clostridium XVIII & major & $0.89 \%$ & $0.33 \%$ \\
Catenibacterium & major & $0.38 \%$ & $0.05 \%$ \\
Phascolarctobacterium & major & $1.47 \%$ & $0.80 \%$ \\
Dialister & major & $0.61 \%$ & $1.51 \%$ \\
Megasphaera & major & $0.55 \%$ & $0.14 \%$ \\
Mitsuokella & major & $0.13 \%$ & $0.27 \%$ \\
Parasutterella & major & $0.15 \%$ & $0.77 \%$ \\
Sutterella & major & $0.38 \%$ & $0.23 \%$ \\
Desulfovibrio & major & $0.16 \%$ & $0.01 \%$ \\
Escherichia/Shigella & major & $0.56 \%$ & $2.39 \%$ \\
Akkermansia & major & $1.01 \%$ & $0.01 \%$ \\
\hline
\end{tabular}

2

3

4

5

6

7

8

9

10

11

12 


\section{Table 5 (on next page)}

AMOVA analysis result between different groups based on microbiota composition. 
1 Table 5. AMOVA analysis result between different groups based on microbiota 2 composition.

\begin{tabular}{lll}
\hline Group1 & Group2 & P value \\
\hline Normal weight ASD $(n=14)$ & Underweight ASD $(n=5)$ & 0.076 \\
Normal weight ASD $(n=14)$ & Obese ASD $(n=2)$ & 0.991 \\
Normal weight ASD $(n=14)$ & Obesity $(n=21)$ & $0.037^{*}$ \\
Underweight ASD $(n=5)$ & Obese ASD $(n=2)$ & 0.674 \\
Underweight ASD $(n=5)$ & Obesity $(n=21)$ & 0.187 \\
Obese ASD $(n=2)$ & Obesity $(n=21)$ & 0.589 \\
ASD $(n=21)$ & Obesity $(n=21)$ & $0.032^{*}$ \\
\hline
\end{tabular}

3

$* \mathrm{P}$ value $<0.05$

4 
Table 6(on next page)

Significantly different species of gut microbiota between ASD and obesity. 
1 Table 6. Significantly different species of gut microbiota between ASD and obesity.

\begin{tabular}{lrrrc}
\hline species & ASD & Control(Obesity) & p-values & \multicolumn{2}{c}{$\begin{array}{l}\text { Enriched } \\
\text { in }\end{array}$} \\
\hline Blautia faecis & $1.00 \%$ & $0.16 \%$ & $1.27 \mathrm{E}-03$ & ASD \\
Blautia wexlerae & $1.18 \%$ & $0.33 \%$ & $1.14 \mathrm{E}-02$ & ASD \\
Dialister succinatiphilus & $0.02 \%$ & $1.38 \%$ & $3.94 \mathrm{E}-03$ & Obesity \\
Eubacterium eligens & $0.86 \%$ & $0.25 \%$ & $4.52 \mathrm{E}-02$ & ASD \\
Fusobacterium mortiferum & $0.08 \%$ & $2.85 \%$ & $4.64 \mathrm{E}-02$ & Obesity \\
Holdemanella biformis & $0.69 \%$ & $0.02 \%$ & $3.27 \mathrm{E}-02$ & ASD \\
Megamonas funiformis & $0.51 \%$ & $10.35 \%$ & $2.25 \mathrm{E}-03$ & Obesity \\
Phascolarctobacterium succinatutens & $0.68 \%$ & $0.08 \%$ & $6.61 \mathrm{E}-03$ & ASD \\
Ruminococcus faecis & $0.69 \%$ & $0.12 \%$ & $1.88 \mathrm{E}-02$ & ASD \\
Prevotella copri & $25.09 \%$ & $26.61 \%$ & $6.61 \mathrm{E}-01$ & $\square$ \\
\hline
\end{tabular}

2

3

4

5

6

7 
Table 7 (on next page)

Function prediction using PICRUSt based on 16S rRNA gene copy numbers. 
1 Table 7. Function prediction using PICRUSt based on 16S rRNA gene copy numbers.

\begin{tabular}{|c|c|c|c|c|}
\hline Level 1 & Level 2 & pathway & p-value & Enriched in \\
\hline Environmental Information Processing & Membrane Transport & Bacterial secretion system & $1.70 \mathrm{E}-03$ & Obesity \\
\hline Environmental Information Processing & Signaling Molecules and Interaction & Cellular antigens & $2.33 \mathrm{E}-02$ & Obesity \\
\hline Environmental Information Processing & Signaling Molecules and Interaction & Ion channels & $9.92 \mathrm{E}-04$ & Obesity \\
\hline Metabolism & Amino Acid Metabolism & Valine, leucine and isoleucine degradation & $3.70 \mathrm{E}-04$ & Obesity \\
\hline Metabolism & Biosynthesis of Other Secondary Metabolites & Flavone and flavonol biosynthesis & $3.03 \mathrm{E}-02$ & ASD \\
\hline Metabolism & Biosynthesis of Other Secondary Metabolites & Isoquinoline alkaloid biosynthesis & $2.03 \mathrm{E}-03$ & Obesity \\
\hline Metabolism & Biosynthesis of Other Secondary Metabolites & Penicillin and cephalosporin biosynthesis & $4.53 \mathrm{E}-02$ & Obesity \\
\hline Metabolism & Enzyme Families & Protein kinases & $1.47 \mathrm{E}-02$ & ASD \\
\hline Metabolism & Glycan Biosynthesis and Metabolism & Glycosaminoglycan degradation & 4.03E-02 & Obesity \\
\hline Metabolism & Glycan Biosynthesis and Metabolism & Glycosphingolipid biosynthesis - ganglio series & $2.46 \mathrm{E}-02$ & Obesity \\
\hline Metabolism & Glycan Biosynthesis and Metabolism & Lipopolysaccharide biosynthesis & $8.36 \mathrm{E}-04$ & Obesity \\
\hline Metabolism & Glycan Biosynthesis and Metabolism & Lipopolysaccharide biosynthesis proteins & 4.95E-04 & Obesity \\
\hline Metabolism & Lipid Metabolism & Ether lipid metabolism & $8.60 \mathrm{E}-03$ & ASD \\
\hline Metabolism & Lipid Metabolism & Linoleic acid metabolism & $3.37 \mathrm{E}-02$ & ASD \\
\hline Metabolism & Lipid Metabolism & Primary bile acid biosynthesis & $6.39 \mathrm{E}-03$ & ASD \\
\hline Metabolism & Lipid Metabolism & Secondary bile acid biosynthesis & $5.97 \mathrm{E}-03$ & ASD \\
\hline Metabolism & Lipid Metabolism & Steroid hormone biosynthesis & $2.67 \mathrm{E}-02$ & Obesity \\
\hline Metabolism & Metabolism of Cofactors and Vitamins & Riboflavin metabolism & $5.23 \mathrm{E}-03$ & Obesity \\
\hline Metabolism & Metabolism of Cofactors and Vitamins & Ubiquinone and other terpenoid-quinone biosynthesis & $7.45 \mathrm{E}-03$ & Obesity \\
\hline Metabolism & Metabolism of Other Amino Acids & D-Arginine and D-ornithine metabolism & $3.58 \mathrm{E}-03$ & Obesity \\
\hline Metabolism & Metabolism of Other Amino Acids & Glutathione metabolism & $2.96 \mathrm{E}-03$ & Obesity \\
\hline Metabolism & Metabolism of Other Amino Acids & Phosphonate and phosphinate metabolism & $1.41 \mathrm{E}-02$ & ASD \\
\hline Metabolism & Metabolism of Terpenoids and Polyketides & Geraniol degradation & $2.29 \mathrm{E}-03$ & Obesity \\
\hline Metabolism & Metabolism of Terpenoids and Polyketides & Limonene and pinene degradation & $2.18 \mathrm{E}-02$ & Obesity \\
\hline Metabolism & Metabolism of Terpenoids and Polyketides & Tetracycline biosynthesis & $1.39 \mathrm{E}-02$ & ASD \\
\hline Metabolism & Xenobiotics Biodegradation and Metabolism & Atrazine degradation & $5.87 \mathrm{E}-03$ & ASD \\
\hline
\end{tabular}


Metabolism

Metabolism

Metabolism

Metabolism

Metabolism

Metabolism

Metabolism
Xenobiotics Biodegradation and Metabolism

Xenobiotics Biodegradation and Metabolism

Xenobiotics Biodegradation and Metabolism

Xenobiotics Biodegradation and Metabolism

Xenobiotics Biodegradation and Metabolism

Xenobiotics Biodegradation and Metabolism

Xenobiotics Biodegradation and Metabolism
Chloroalkane and chloroalkene degradation

Chlorocyclohexane and chlorobenzene degradation

Dioxin degradation

Ethylbenzene degradation

Styrene degradation

Toluene degradation

Xylene degradation
3.09E-03

2.32E-02

$1.04 \mathrm{E}-03$

$6.98 \mathrm{E}-03$

4.36E-03

3.14E-04

$5.69 \mathrm{E}-04$
ASD

ASD

ASD

Obesity

ASD

Obesity

ASD 
Table 8(on next page)

Correlations of species calculated using Spearman algorithm. 
1 Table 8. Correlations of species calculated using Spearman algorithm.

\begin{tabular}{|c|c|c|c|}
\hline$\square$ & difference species & correlated species & spearmanCoef \\
\hline \multirow{10}{*}{ ASD } & Holdemanella biformis & Bacteroides plebeius & $.537^{*}$ \\
\hline & Blautia wexlerae & Bacteroides vulgatus & $.509^{*}$ \\
\hline & Dialister succinatiphilus & Bacteroides vulgatus & $.479^{*}$ \\
\hline & Phascolarctobacterium succinatutens & Bacteroides vulgatus & $-.632^{* *}$ \\
\hline & Blautia wexlerae & Blautia faecis & $.702^{* *}$ \\
\hline & Dialister succinatiphilus & Blautia faecis & $.459^{*}$ \\
\hline & Fusobacterium mortiferum & Prevotella copri & $.550^{* *}$ \\
\hline & Holdemanella biformis & Prevotella copri & $.470^{*}$ \\
\hline & Megamonas funiformis & Prevotella copri & $.446^{*}$ \\
\hline & Phascolarctobacterium succinatutens & Prevotella copri & $.609^{* *}$ \\
\hline \multirow{17}{*}{$\begin{array}{c}\text { Control(Obesity } \\
\text { ) }\end{array}$} & Megamonas funiformis & Bacteroides vulgatus & $-.588^{* *}$ \\
\hline & Blautia faecis & Roseburia faecis & $.608^{* *}$ \\
\hline & Blautia faecis & $\begin{array}{l}\text { Faecalibacterium } \\
\text { prausnitzii }\end{array}$ & $.544^{*}$ \\
\hline & Blautia faecis & Eubacterium eligens & $.481^{*}$ \\
\hline & Blautia wexlerae & Bacteroides vulgatus & $.465^{*}$ \\
\hline & Blautia wexlerae & Roseburia faecis & $.558^{* *}$ \\
\hline & Blautia wexlerae & Blautia faecis & $.464^{*}$ \\
\hline & Blautia wexlerae & Eubacterium eligens & $.439^{*}$ \\
\hline & Blautia wexlerae & Ruminococcus faecis & $.477^{*}$ \\
\hline & Eubacterium eligens & Roseburia faecis & $.850^{* *}$ \\
\hline & Eubacterium eligens & Streptococcus salivarius & $.529^{*}$ \\
\hline & Fusobacterium mortiferum & $\begin{array}{l}\text { Faecalibacterium } \\
\text { prausnitzii }\end{array}$ & $-.594^{* *}$ \\
\hline & Fusobacterium mortiferum & Dialister succinatiphilus & $-.511^{*}$ \\
\hline & Fusobacterium mortiferum & Escherichia coli & $.504^{*}$ \\
\hline & Megamonas funiformis & $\begin{array}{l}\text { Faecalibacterium } \\
\text { prausnitzii }\end{array}$ & $-.514^{*}$ \\
\hline & Megamonas funiformis & Blautia wexlerae & $-.600^{* *}$ \\
\hline & Megamonas funiformis & Fusobacterium mortiferum & $.515^{*}$ \\
\hline
\end{tabular}


Table 9 (on next page)

Significantly different taxa between normal weight ASD and Obesity in addition to all ASD and Obesity. 
Table 9. Significantly different taxa between normal weight ASD and Obesity in addition to all ASD and Obesity.

\begin{tabular}{|c|c|c|c|c|c|c|}
\hline \multirow[b]{2}{*}{ Taxonomy } & \multirow{2}{*}{$\begin{array}{c}\text { p-values between } \\
\text { normal ASD and } \\
\text { Obesity }\end{array}$} & \multicolumn{3}{|c|}{ Abundance (\%) } & \multirow{2}{*}{$\begin{array}{c}\text { p-values between } \\
\text { all ASD and } \\
\text { Obesity }\end{array}$} & \multirow{2}{*}{$\begin{array}{l}\square \\
\square \\
\square\end{array}$} \\
\hline & & $\begin{array}{l}\text { normal ASD } \\
(n=14)\end{array}$ & $\begin{array}{l}\text { Obesity } \\
(n=21)\end{array}$ & $\begin{array}{l}\text { all ASD } \\
(\mathrm{n}=\mathbf{2 1})\end{array}$ & & \\
\hline Firmicutes & 0.0118 & 54.23 & 37.55 & 49.74 & 0.0518 & phylum \\
\hline Bacteroidetes & 0.0255 & 37.26 & 53.44 & 43.56 & 0.1503 & phylum \\
\hline Fusobacteria & 0.0324 & 0.13 & 3.52 & 0.10 & 0.0308 & phylum \\
\hline Desulfovibrionaceae & 0.0333 & 0.37 & 0.03 & 0.35 & 0.0105 & family \\
\hline Erysipelotrichaceae & 0.0007 & 3.4 & 0.52 & 2.71 & 0.0003 & family \\
\hline Fusobacteriaceae & 0.0317 & 0.12 & 3.51 & 0.09 & 0.0304 & family \\
\hline Lachnospiraceae & 0.0038 & 29.29 & 12.42 & 25.89 & 0.0046 & family \\
\hline Porphyromonadaceae & 0.0113 & 1.28 & 0.47 & 1.01 & 0.028 & family \\
\hline Ruminococcaceae & 0.0218 & 12.33 & 5.68 & 11.91 & 0.0125 & family \\
\hline Veillonellaceae & 0.0004 & 1.37 & 13.95 & 2.09 & 0.0007 & family \\
\hline Bilophila & 0.024 & 0.19 & 0.02 & 0.15 & 0.0146 & genus \\
\hline Blautia & 0.0097 & 3.65 & 1.08 & 3.283 & 0.0034 & genus \\
\hline Clostridium IV & 0.0209 & 1.38 & 0.1 & 1.04 & 0.0097 & genus \\
\hline Clostridium XlVa & 0.0137 & 1.74 & 0.65 & 1.44 & 0.0082 & genus \\
\hline Fusobacterium & 0.0355 & 0.1 & 3.17 & 0.08 & 0.0343 & genus \\
\hline Holdemanella & 0.0225 & 1.39 & 0.03 & 1.08 & 0.0075 & genus \\
\hline Holdemania & 0.0227 & 0.005 & 0.001 & 0.005 & 0.0047 & genus \\
\hline Howardella & 0.1026 & 0.028 & 0.002 & 0.03 & 0.0431 & genus \\
\hline Intestinibacter & 0.0573 & 0.03 & 0.002 & 0.02 & 0.0444 & genus \\
\hline Intestinimonas & 0.086 & 0.01 & 0.002 & 0.02 & 0.0263 & genus \\
\hline Lachnospiracea_incertae_sedis & 0.0025 & 5.19 & 1.69 & 4.28 & 0.0054 & genus \\
\hline Megamonas & 0.0016 & 0.52 & 11.77 & 0.7 & 0.0018 & genus \\
\hline Murimonas & 0.1054 & 0.0007 & 0.0001 & 0.0008 & 0.0437 & genus \\
\hline Odoribacter & 0.0333 & 0.07 & 0.01 & 0.05 & 0.0197 & genus \\
\hline
\end{tabular}




\begin{tabular}{|c|c|c|c|c|c|c|}
\hline Oscillibacter & 0.002 & 0.3 & 0.07 & 0.26 & 0.0015 & genus \\
\hline Ruminococcus & 0.0663 & 2.76 & 0.37 & 2.81 & 0.0066 & genus \\
\hline Ruminococcus 2 & 0.0292 & 1.46 & 0.26 & 1.14 & 0.0165 & genus \\
\hline Senegalimassilia & 0.0344 & 0.03 & 0.002 & 0.03 & 0.0144 & genus \\
\hline Terrisporobacter & 0.0282 & 0.02 & 0.006 & 0.02 & 0.0303 & genus \\
\hline Turicibacter & 0.0457 & 0.21 & 0.02 & 0.16 & 0.0402 & genus \\
\hline Allisonella & 0.0298 & 0.004 & 0.04 & 0.009 & 0.0552 & genus \\
\hline Bacteroides plebeius & 0.0233 & 0.17 & 5.59 & 1.68 & 0.1571 & species \\
\hline Blautia faecis & 0.0023 & 0.94 & 0.16 & 1 & 0.0013 & species \\
\hline Blautia wexlerae & 0.0229 & 1.42 & 0.33 & 1.18 & 0.0114 & species \\
\hline Dialister succinatiphilus & 0.0037 & 0.01 & 1.38 & 0.02 & 0.0039 & species \\
\hline Eubacterium eligens & 0.0608 & 0.95 & 0.25 & 0.86 & 0.0452 & species \\
\hline Fusobacterium mortiferum & 0.0479 & 0.09 & 2.85 & 0.08 & 0.0464 & species \\
\hline Holdemanella biformis & 0.0629 & 0.88 & 0.02 & 0.69 & 0.0327 & species \\
\hline Megamonas funiformis & 0.002 & 0.37 & 10.35 & 0.51 & 0.0022 & species \\
\hline $\begin{array}{l}\text { Phascolarctobacterium } \\
\text { succinatutens }\end{array}$ & 0.0253 & 0.57 & 0.08 & 0.68 & 0.0066 & species \\
\hline Ruminococcus faecis & 0.0399 & 0.86 & 0.12 & 0.69 & 0.0188 & species \\
\hline
\end{tabular}

